\title{
ON THE HOMOTOPY INDEX FOR INFINITE-DIMENSIONAL SEMIFLOWS ${ }^{1}$
}

BY

\author{
KRZYSZTOF P. RYBAKOWSKI ${ }^{2}$
}

\begin{abstract}
In this paper we consider semiflows whose solution operator is eventually a conditional $\alpha$-contraction. Such semiflows include solutions of retarded and neutral functional differential equations, of parabolic and certain other classes of partial differential equations. We prove existence of (nonsmooth) isolating blocks and index pairs for such semiflows, via the construction of special Lyapunov functionals. We show that index pairs enjoy all the properties needed to define the notion of a homotopy index, thus generalizing earlier results of Conley [2]. Finally, using a result of Mañé [9], we prove that, under additional smoothness assumptions on the semiflow, the homotopy index is essentially a finite-dimensional concept. This gives a formal justification of the applicability of Wazewski's Principle to infinite-dimensional problems. Several examples illustrate the theory.
\end{abstract}

Introduction. If $\pi$ is a semiflow on a topological space $X$, then an isolating block $B$ is a closed subset of $X$ all of whose boundary points are well behaved with respect to $\pi$. In particular, every trajectory starting inside of $B$ and reaching the boundary of $B$ at some positive (resp. negative) time $t_{0}$ is required to leave $B$ immediately as we move forward (resp. backward) of $t_{0}$. (See $\$ 2$ for the definition of an isolating block.)

If $\pi$ is a (two-sided) flow on a locally compact set $X$, if $K$ is a nonempty, compact and isolated invariant set and $N$ is an isolating neighborhood of $K$, then it was proved in [1], [3] and [13] that there is an isolating block $B$ such that $K \subset B \subset N$. This paper proves an analogue of this statement in the context of (one-sided) local semiflows $\pi$ on a not necessarily locally compact metric space $X$. Although a certain compactness condition (H2) is imposed on $\pi$, it is satisfied, e.g., if $X$ is a Banach space and the map $x \rightarrow x \pi t_{0}$ is a conditional $\alpha$-contraction for some $t_{0}>0$. Hence the result can be applied to RFDE's, to NFDE's with stable difference operators, to parabolic PDE's as well as certain other classes of equations.

Our proof of the existence of isolating blocks is similar in spirit to the one of Wilson and Yorke [13], where Lyapunov functions are used extensively. One of the primary difficulties in our case arises with the determination of a Lyapunov function whose level curves are transversal to the stable manifold of the invariant set. This requires several new ideas which may be considered an important contribution of this paper.

Received by the editors December 18, 1980 .

1980 Mathematics Subject Classification. Primary 47H09, 55P10.

${ }_{1}^{1}$ This research was supported by the Deutsche Forschungsgemeinschaft.

2 Visiting Assistant Professor (Research) at Brown University.

(C) 1982 American Mathematical Society 0002-9947/82/0000-0759/\$09.00 
If $B$ is an isolating block and if $B^{+}$is the set of all points $x \in \partial B$ such that the trajectory through $x$ leaves $B$ immediately in positive direction, then $\left(B, B^{+}\right)$is an example of an index pair (see $\$ 3$ for a definition). Index pairs $\left(N_{1}, N_{2}\right)$ were introduced by Conley [2]. Their existence and several important properties including the construction of the homotopy index are known for (one-sided) semiflows $\pi$ on a locally compact space $X$ (see [2]).

The homotopy index of [2] is an important tool in the study of the qualitative behavior of semiflows. Its formal similarity with the Brouwer topological degree raises the question, if the homotopy index can be generalized to semiflows satisfying certain $\alpha$-contraction hypotheses, analogously to the Nussbaum fixed point index. It is proved in this paper that this is, indeed, the case. By combining ideas from [2] with the method of Lyapunov functions used in the existence proof of isolating blocks, we prove the existence and all the important properties leading to the concept of a homotopy index for semiflows $\pi$ satisfying the hypothesis (H2). In this way, Lyapunov functions become a unifying technique in the construction of both isolating blocks and index pairs.

Since an isolating block satisfies the continuity assumption of the principle of Ważewski, the existence theorem for isolating blocks can be regarded as a partial converse of Ważewski's Theorem.

Of course, even for scalar ODE's, like $\dot{x}=|x|$, the retract assumptions of Ważewski's Theorem are not necessarily satisfied.

On the other hand, classical examples suggest that even if we work in an infinite-dimensional space, say $C\left([-r, 0], \mathbf{R}^{n}\right)$, and, therefore, the general retract behavior is different than in finite dimensions, this will not affect the applicability of Ważewski's Principle, since the sets satisfying Ważewski's continuity hypothesis seem to behave like finite-dimensional sets. This is made more precise in $\$ 4$, where it is shown that, under certain additional smoothness assumptions, for every index pair $\left(N_{1}, N_{2}\right)$ there exists a compact finite-dimensional set whose AlexanderSpanier cohomology module is isomorphic to that of $\left(N_{1}, N_{2}\right)$. In particular, $\bar{H}^{q}\left(N_{1}, N_{2} ; G\right)=\bar{H}^{q}\left(N_{1} / N_{2} ; G\right)=0$ for all sufficiently large $q$ 's.

1. Notation, preliminaries. If $X$ is a topological space, and $A \subset X$, then $\mathrm{Cl} A$, Int $A, \partial A$ denote the closure, the interior, and the boundary of $A$ (in $X$ ), respectively. If $X$ is a metric space, then $d(x, y)$ is the distance between $x$ and $y . \mathbf{R}^{+}\left(\mathbf{R}^{-}\right)$ denotes the set of nonnegative (resp. nonpositive) reals.

Let $\Omega$ be an open set in $\mathbf{R}^{n}$. If $\alpha$ is a multiindex, $\alpha=\left(\alpha_{1}, \ldots, \alpha_{n}\right), \alpha_{k}$ nonnegative integers, we write $|\alpha|=\alpha_{1}+\cdots+\alpha_{n}, D^{\alpha}:=\partial^{|\alpha|} / \partial x_{\alpha_{1}} \cdots \cdot \partial x_{\alpha_{n}}$. We use the following standard symbols:

$L^{2}(\Omega)$ is the Hilbert space of equivalence classes of Lebesgue-square integrable complex valued functions on $\Omega$ with the scalar product.

$(f, g)=\int_{\Omega} f(x) \cdot \bar{g}(x) d \lambda^{n}(x) .\left(\lambda^{n}\right.$ denotes the Lebesgue measure on $\mathbf{R}^{n}$.)

$\hat{C}^{m}(\Omega)$ is the normed space of all $m$-times continuously differentiable complex valued functions $u$ on $\Omega$ for which the following norm is finite:

$$
\|u\|_{m}=\left(\sum_{|\alpha|<m} \int_{\Omega}\left|D^{\alpha} u(x)\right|^{2} d \lambda^{n}(x)\right)^{1 / 2} \text {. }
$$


$\hat{C}_{0}^{m}(\Omega)$ is the subset of $\hat{C}^{m}(\Omega)$ consisting of functions with compact support in $\Omega$. $H^{m}(\Omega), H_{0}^{m}(\Omega)$ are completions of $\hat{C}^{m}(\Omega)$ and $\hat{C}_{0}^{m}(\Omega)$ resp., with respect to the norm $\|u\|_{m}$.

If $A$ is an operator in a Banach space, then $\rho(A), \sigma(A)$ are the resolvent set of $A$ and the spectrum of $A$, respectively.

Definition 1.1 (CF. [14]). The pair $(X, \pi)$ is called a local semiflow (on $X$ ), if the following properties are satisfied:

(1) $X$ is a topological space, $\pi: D \rightarrow X$ is a continuous mapping, $D$ being an open subset of $\mathbf{R}^{+} \times X$.

(We write $x \pi t$ for $\pi(t, x)$.)

(2) For every $x \in X$ there is an $\omega_{x}, 0<\omega_{x} \leqslant \infty$, such that $(t, x) \in D$ if and only if $0 \leqslant t<\omega_{x}$.

(3) $x \pi 0=x$ for $x \in X$.

(4) If $(t, x) \in D$ and $(s, x \pi t) \in D$, then $(t+s, x) \in D$ and $x \pi(t+s)=(x \pi t) \pi s$.

REMARKS. 1. If $\omega_{x}=\infty$ for every $x \in X$, then the local semiflow $(X, \pi)$ is simply called a semiflow (on $X$ ).

2. In the sequel, we shall frequently omit the mention of $X$ and call $\pi$ a (local) semiflow.

Definition 1.2. Let $(X, \pi)$ be a local semiflow. Let $x \in X$, and $\sigma: I \rightarrow X$ be a mapping, where $I=[a, 0],-\infty<a<0$, or $I=(a, 0],-\infty \leqslant a<0$. $\sigma$ is called a left solution through $x$, if $\sigma(0)=x$, and for all $t \in I, s \in \mathbf{R}^{+}$for which $t+s \leqslant 0$, it follows that $s<\omega_{\sigma(t)}$ and $\sigma(t) \pi s=\sigma(t+s)$.

If $Y$ is a subset of $X$, then set:

$A^{+}(Y)=\left\{x \in X \mid x \pi\left[0, \omega_{x}\right) \subset Y\right\}$,

$A^{-}(Y)=\left\{x \in X \mid\right.$ there is a left solution $\sigma$ through $x$, defined on $\mathbf{R}^{-}$, such that $\left.\sigma\left[\mathbf{R}^{-}\right] \subset Y\right\}$.

$Y$ is called positively invariant iff $Y=A^{+}(Y)$.

$Y$ is called negatively invariant iff $Y=A^{-}(Y)$.

$Y$ is called invariant iff $Y$ is both positively and negatively invariant.

Unless otherwise specified, $(X, \pi)$ is a fixed local semiflow on a metric space $X$.

Let $\langle K, U, N\rangle$ be a triple of sets in $X$. We shall introduce the following hypotheses (H1)-(H3) about $\langle K, U, N\rangle$ :

(H1) $K \subset U \subset \mathrm{Cl} U=N, U$ is open, $K$ is closed, and $K$ is the largest invariant set in $N$.

(H2) If $x_{n} \in N, 0 \leqslant t_{n}<\omega_{x}, x_{n} \pi\left[0, t_{n}\right] \subset N$, and $t_{n} \rightarrow \infty$, then the sequence $\left\{x_{n} \pi t_{n}\right\}$ is precompact (i.e. relatively compact).

(H3) If $x \in N$ and $\omega_{x}<\infty$, then there is a $t, 0 \leqslant t<\omega_{x}$, such that $x \pi t \notin N$.

REMARKs. 1. If $N$ is a closed subset of $X$ such that the largest invariant set $K$ in $N$ is disjoint from the boundary of $N$ (i.e. $K \cap \partial N=\varnothing$ ), then $N$ is called an isolating neighborhood (of $K$ ). If, on the other hand, $K$ is a closed invariant set and there is a neighborhood $U$ of $K$ such that $K$ is the largest invariant set in $U$, then $K$ is called an isolated invariant set. Hence, if $\langle K, U, N\rangle$ satisfy $(\mathrm{H} 1)$, then $K$ is an isolated invariant set and $N$ is an isolating neighborhood of $K$.

2. If $\omega_{x}=\infty$ for $x \in X$, i.e. if $\pi$ is a semiflow, then (H3) is vacuously satisfied. In the general case, (H3) simply means that no trajectory blows up in $N$. 
3. Let $E$ be a Banach space, $N \subset X \subset E, N$ bounded and closed, and let $\alpha$ be the Kuratowski measure of noncompactness on $E$. A mapping $T: D(T) \rightarrow E$, $D(T) \subset E$, is called a conditional $\alpha$-contraction, if there is a constant $k, 0<k<1$, such that whenever $B$ is a bounded subset of $D(T)$ for which $T(B)$ is bounded, then $\alpha(T(B)) \leqslant k \alpha(B)$. If the mapping $x \rightarrow x \pi t_{0}$ (defined for all $x$ such that $\left.t_{0}<\omega_{x}\right)$ is a conditional $\alpha$-contraction for some $t_{0}>0$, then $(\mathrm{H} 2)$ is satisfied for $\pi$ and $N$.

4. If (H2) is satisfied for the closed set $N$, then $N$ is an isolating neighborhood if and only if there is a $t_{0}>0$ such that whenever $x \pi 2 t_{0}$ is defined and $x \pi\left[0,2 t_{0}\right] \subset$ $N$, then $x \pi t_{0} \notin \partial N$.

LEMMA 1.1. Let $(\mathrm{H} 1)-(\mathrm{H} 3)$ be satisfied. Then the following properties hold:

(i) $A^{-}(N)$ is compact, $K$ is compact.

(ii) If $\left\{x_{n}\right\},\left\{t_{n}\right\}$ satisfy (H2), then every cluster point of $\left\{x_{n} \pi t_{n}\right\}$ belongs to $A^{-}(N)$.

(iii) Let $x_{n} \in N, 0 \leqslant t_{n}<\infty, x_{n} \pi\left[0, t_{n}\right] \subset N$, and $x_{n} \rightarrow x_{0}$.

Then:

(1) $t_{n} \rightarrow t_{0}$ and $t_{0}<\infty$ imply that $t_{0}<\omega_{x_{0}}$ and $x_{n} \pi t_{n} \rightarrow x_{0} \pi t_{0}$ and $x_{0} \pi\left[0, t_{0}\right] \subset N$.

(2) $t_{n} \rightarrow \infty$ implies that $x_{0} \in A^{+}(N)$.

The proof of the lemma is left to the reader as an easy exercise.

EXAMPLE 1.1 (CF. [6]). Let $C=C\left([-r, 0], \mathbf{R}^{n}\right)$ be the space of continuous functions from [ $-r, 0$ ] into $\mathbf{R}^{n}$ equipped with the sup-norm topology. If $t \in \mathbf{R}$, and $x:[-r+t, t] \rightarrow \mathbf{R}^{n}$ is a continuous mapping, then $x_{t} \in C$ is defined as $x_{t}(\theta)=$ $x(t+\theta), \theta \in[-r, 0]$.

Now let $\Omega$ be open in $C, f: \Omega \rightarrow \mathbf{R}^{n}$ be continuous, and $D: C \rightarrow \mathbf{R}^{n}$ be linear and bounded. Then there is a mapping $\eta:[-r, 0] \rightarrow L\left(\mathbf{R}^{n}, \mathbf{R}^{n}\right), \eta$ of bounded variation, such that for every $\phi \in C, D \phi=\int_{-r}^{0} d_{\theta} \eta \cdot \phi(\theta)$. D is called a stable difference operator, if (1) and (2) below hold:

(1) $D$ is atomic at zero, i.e., $\eta(0)-\lim _{\theta \rightarrow 0^{-}} \eta(\theta)$ is nonsingular.

There are constants $a, b>0$, such that for every $h \in C\left(\mathbf{R}^{+}, \mathbf{R}^{n}\right)$ and every solution $y:[-r, \infty) \rightarrow \mathbf{R}^{n}$ of the equation $D y_{t}=h(t), t \geqslant 0$, it follows that

$$
\left|y_{t}\right| \leqslant b e^{-a t}\left|y_{0}\right|+b \sup _{0 \leqslant u<t}|h(u)| \text {. }
$$

Consider the equation (1.1):

$$
d D\left(x_{t}\right) / d t=f\left(x_{t}\right)
$$

Equation (1.1) is called a neutral functional differential equation (NFDE). A continuous function $x:[-r, A) \rightarrow \mathbf{R}^{n}, A>0$, is said to be a solution of (1.1) through $\phi \in C$ if the function $t \rightarrow D\left(x_{t}\right)$ is continuously differentiable on $[0, A),(1.1)$ is satisfied for $t \in[0, A)$, and $x_{0}=\varphi$.

If $D$ has the special form $D \phi=\phi(0)$, then the resulting NFDE takes the form

$$
\dot{x}(t)=f\left(x_{t}\right)
$$

and is called a retarded functional differential equation (RFDE).

Suppose that for every $\phi \in \Omega$ there is a unique solution $x(\phi)$ of (1.1) through (1.2). Denote $T(t) \phi=x_{t}(\phi)$. Writing $\phi \pi t$ for $T(t) \phi$ we obtain a local semiflow on 
$X=\Omega$, which we shall call the local semiflow generated by a NFDE (or, in the special case of (1.2), by $a$ RFDE).

Now suppose that $N$ is a closed bounded set in $\Omega$ on which $f$ is bounded. Then Theorem 2.5 on p. 279 in [6] implies that (H3) is satisfied. To prove (H2) it is enough to notice that, by Corollary 6.2, p. 296 in [6], the solution operator $T(t)$ is a conditional $\alpha$-contraction with respect to some equivalent norm on $C$, and then apply our previous remarks. In view of some later applications, we give a direct proof of (H2). Let $\phi_{n}, t_{n}$ be such that $\phi_{n} \pi\left[0, t_{n}\right] \subset N$ and $t_{n} \rightarrow \infty$. We may assume $t_{n} \geqslant r$. To prove that $\left\{\phi_{n} \pi t_{n}\right\}$ is precompact, it suffices to show that $\left\{\phi_{n} \pi\left(t_{n}-r\right)\right\}$ is equicontinuous. Let $\varepsilon>0$ be arbitrary, choose $r>\delta>0$ such that $M b \delta<\varepsilon / 2$. (If $r=0$, then $\left\{\phi_{n} \pi t_{n}\right\}$ is obviously precompact.) Choose $t_{0}$ such that $2 A b e^{-a t_{0}}<$ $\varepsilon / 2$. Here, $M=\sup _{x \in N}|f(x)|, A=\sup _{x \in N}|x|$. Now there is an $n_{0}=n_{0}\left(\varepsilon, t_{0}\right)$ such that $t_{n}-r \geqslant t_{0}$ for $n>n_{0}$. Let $0<\delta_{1}<\delta$ be such that for all $n, 1 \leqslant n \leqslant n_{0}$, all $\theta \in[-r, 0]$, and $|\tau|<\delta_{1}$, it follows that $\left|\left(\phi_{n} \pi\left(t_{n}-r\right)\right)(\theta+\tau)-\left(\phi_{n} \pi\left(t_{n}-r\right)\right)(\theta)\right|$ $<\varepsilon$, whenever $\theta+\tau \in[-r, 0]$. Fix $\tau,|\tau|<\delta_{1}$. For $n>n_{0}$, define

$$
\begin{aligned}
& y^{n}(t)=x\left(\phi_{n}\right)(t+\tau)-x\left(\phi_{n}\right)(t), \quad \text { for } t \leqslant t_{n}-r, \\
& y^{n}(t)=y^{n}\left(t_{n}-r\right), \text { for } t>t_{n}-r .
\end{aligned}
$$

Set $h^{n}(t)=D y_{t}{ }^{n}$. Then $h^{n} \in C\left(\mathbf{R}^{+}, \mathbf{R}^{n}\right)$. Hence

$$
\left|y_{t_{n}-r}^{n}\right| \leqslant b e^{-a t_{0}}\left|y_{0}\right|+b \sup _{0<u<t_{n}-r}\left|h^{n}(u)\right| .
$$

It follows for all $n>n_{0}$ and $\theta \in[-r, 0], \theta+\tau \leqslant 0$, that

$$
\begin{aligned}
\mid\left(\phi_{n} \pi\left(t_{n}-r\right)\right) & (\theta+\tau)-\left(\phi_{n} \pi\left(t_{n}-r\right)\right)(\theta) \mid \\
& =\left|x\left(\phi_{n}\right)\left(t_{n}-r+\theta+\tau\right)-x\left(\phi_{n}\right)\left(t_{n}-r+\theta\right)\right| \\
& =\left|y_{t_{n}-r}^{n}(\theta)\right| \leqslant 2 A b e^{-a t_{0}}+b \sup _{0<u<t_{n}-r} \int_{u}^{u+\tau}\left|f\left(x_{s}\left(\phi_{n}\right)\right)\right| d u \\
& <\varepsilon / 2+b M|\tau|<\varepsilon / 2+b M \delta<\varepsilon .
\end{aligned}
$$

This immediately implies that $\left\{\phi_{n} \pi\left(t_{n}-r\right)\right\}$ is equicontinuous. Hence (H2) is satisfied.

EXAMPLE 1.2 (CF. [5] AND [7]). Let $X$ be a Banach space, and $A: D(A) \rightarrow X$ be a closed, densely defined, linear operator in $X . A$ is called sectorial, if there are constants $\phi, M, a, 0<\phi<\pi / 2, M \geqslant 1, a \in \mathbf{R}$ such that the sector $S_{\phi, a}=\{\lambda \in$ $C|\lambda \neq a, \phi<\arg | \lambda-a \mid \leqslant \pi\}$ is contained in $\rho(A)$, the resolvent set of $A$, and $\left\|(\lambda-A)^{-1}\right\| \leqslant M /|\lambda-a|$ for all $\lambda \in S_{\phi, a}$. If $A$ is sectorial, then there is a $k>0$ such that $\operatorname{Re} \sigma(A+k I)>0$. Let $A_{1}=A+k I$. For $0<\alpha<1$ define

$$
A_{1}^{-\alpha}=\frac{\sin \pi \alpha}{\pi} \int_{0}^{\infty} \lambda^{-\alpha}\left(\lambda+A_{1}\right)^{-1} d \lambda .
$$

$A_{1}^{-\alpha}$ is bounded and injective. Let $X^{\alpha}$ be the range of $A_{1}^{-\alpha}, X^{0}=X, X^{1}=D(A)$. Let $A_{1}^{\alpha}: X^{\alpha} \rightarrow X$ be the inverse of $A_{1}^{-\alpha}, A^{0}=\mathrm{Id}_{X}, A^{1}=A . X^{\alpha}$ is dense in $X$. Define the norm \|\|$_{\alpha}$ on $X^{\alpha}$ by $\|u\|_{\alpha}=\left\|A_{1}^{\alpha} u\right\|$, where \|\| is the norm for $X . X^{\alpha}$ does not depend on the choice of $k$, and different choices of $k$ yield equivalent norms on $X^{\alpha} . X^{\alpha}$ is a Banach space under $\|\cdot\|_{\alpha}$. 
Suppose $0 \leqslant \alpha<1, U$ is open in $X^{\alpha}$ and $f: U \rightarrow X$ is a locally Lipschitz continuous mapping. Consider the equation (1.3)

$$
d u / d t+A u=f(u) .
$$

Let $u_{0} \in U$. By a solution of $(1.3)$ on $(0, A)$ through $u_{0}$ we mean a continuous mapping $u:[0, A) \rightarrow U$, such that $u(0)=u_{0}, u$ is differentiable on $(0, A), u(t) \in$ $D(A)$ for $t \in(0, A), t \rightarrow f(u(t))$ is locally Hölder continuous, $\int_{0}^{a}\|f(u(t))\| d t<\infty$ for some $a>0$, and (1.2) holds for $t \in(0, A)$. In this definition, " $t \rightarrow g(t)$ is locally Hölder-continuous" means that for every $t_{0}$ there exist a neighborhood $W$ of $t_{0}$ and $L, \theta>0$ such that $\left\|g\left(t_{1}\right)-g\left(t_{2}\right)\right\| \leqslant L\left|t_{1}-t_{2}\right|^{\theta}$ for $t_{1}, t_{2} \in W$.

It follows from [5] that, under the above assumptions, for every $u_{0} \in U$ there exists a unique solution $u\left(u_{0}\right)(t)$ of (1.3) through $u_{0}$, defined on a maximal interval $\left[0, \omega_{u_{0}}\right)$. Defining $u_{0} \pi t=u\left(u_{0}\right)(t)$ for $t<\omega_{u_{0}}$, we obtain a local semidynamical system. If $N$ is a closed bounded subset of $U$ on which $f$ is bounded, then (H3) is satisfied for $N$. If, in addition, $R(\lambda ; A)=(\lambda-A)^{-1}$ is compact for some, and hence every $\lambda \in \rho(A)$, then (H2) is also satisfied for $N$.

The most important example of a sectorial operator arises in the following way: Let $\Omega$ be an open, bounded set in $\mathbf{R}^{n}$ whose boundary is of class $C^{2 m}$ ( $m$ an integer). Let $X=L^{2}(\Omega), \quad D(A)=H^{2 m}(\Omega) \cap H_{0}^{m}(\Omega), \quad(A u)(x):=\sum_{|\alpha|<m} a_{\alpha}(x)$. $D^{\alpha} u(x)$, where the $a_{\alpha}: \mathrm{Cl} \Omega \rightarrow C$ are continuous mappings, and $D^{\alpha} u$ is understood in the distributional sense. Suppose that $A$ is uniformly strongly elliptic on $\Omega$, i.e., there is a $c_{0}>0$ such that $(-1)^{m} \operatorname{Re}\left\{\Sigma_{|\alpha|=m} a_{\alpha}(x) \cdot \xi^{\alpha}\right\}>c_{0}|\xi|^{2 m}$ for all $\xi=$ $\left(\xi_{\alpha}\right)_{|\alpha|<m}, \xi_{\alpha} \in \mathbf{R}$, and all $x \in \Omega$. Then equation (1.3) is called a semilinear parabolic PDE. Results in [5], [7] imply that $A$ is sectorial, and $R(\lambda, A)$ is compact for every $\lambda \in \rho(A)$. Hence it follows that if $f$ is as above, then the solutions of the PDE (1.3) generate a local semiflow on $U$, satisfying (H2) and (H3) for every closed bounded set $N$ on which $f$ is bounded.

2. Isolating blocks. In this section, we shall introduce the concept of an isolating block and prove a main existence theorem.

Definition 2.1. Let $(X, \pi)$ be a local semiflow on the topological space $X$. Let $B$ be a closed subset of $X$, and let $x \in \partial B$.

$x$ is called a strict egress (resp. strict ingress) point (with respect to $\pi$ ) if (1) and (2) below hold:

(1) There is an $h, 0<h<\omega_{x}$, such that $x \pi t \notin B$ (resp. $x \pi t \in$ Int $B$ ) for $t \in(0, h]$.

(2) For every left solution $\sigma$ through $x$, there is an $h<0$, such that $\sigma(t)$ is defined and $\sigma(t) \in \operatorname{Int} B$ (resp. $\sigma(t) \notin B$ ) for $t \in[h, 0)$.

$x$ is called a bounce-off point (with respect to $\pi$ ), if (1) and (2) below hold:

(1) There is an $h, 0<h<\omega_{x}$, such that $x \pi t \notin B$ for $t \in(0, h]$.

(2) For every left solution $\sigma$ through $x$, there is an $h<0$ such that $\sigma(t)$ is defined and $\sigma(t) \notin B$ for $t \in[h, 0)$.

$B$ is called an isolating block (with respect to $\pi$ ) if every boundary point of $B$ is either a strict egress of a strict ingress or a bounce-off point (with respect to $\pi$ ). 


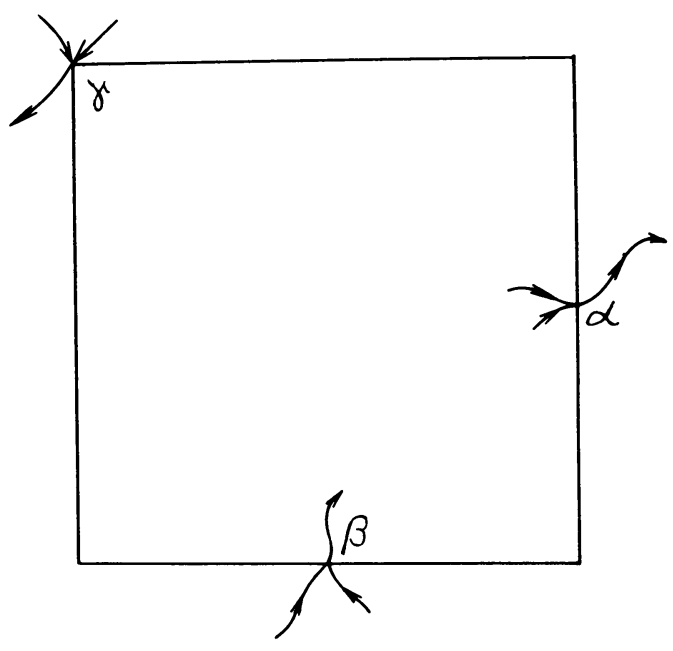

Figure 1

In Figure 1, $\alpha, \beta$ and $\gamma$ are, respectively, a strict egress, and a bounce-off point.

In the sequel, $b^{+}, b^{-}$, and $\tau$ denote the set of strict egress, strict ingress and bounce-off points of $B$, respectively. Note that $\tau$ and $b^{+}$are not necessarily disjoint.

Proposition 2.1. If $B$ is an isolating block, then $b^{+} \cup \tau$ is a strong deformation retract of $B \backslash A^{+}(B)$.

Let us recall that a subset $Y$ of a topological space $Z$ is called a strong deformation retract of $Z$ if there exists a continuous mapping $F: Z \times[0,1] \rightarrow Z$ such that:

(i) $F(z, 0)=z$ for $z \in Z$,

(ii) $F(z, \alpha)=z$ for $z \in Y, \alpha \in[0,1]$,

(iii) $F(z, 1) \in Y$ for $z \in Z$.

To prove Proposition 2.1, let $s^{+}: B \rightarrow \mathbf{R}^{+} \cup\{\infty\}$ is defined as $s^{+}(x)=\sup \{t \mid t$ $\left.<\omega_{x}, x \pi[0, t] \subset B\right\}$. Set $F(x, \alpha)=x \pi\left(\alpha \cdot s^{+}(x)\right), \quad x \in B \backslash A^{+}(B), \alpha \in[0,1]$. Standard arguments prove that $F$ satisfies all properties required.

Hence, as in the classical case of flows on compact sets, isolating blocks permit the use of Ważewski method. In fact, if $b^{+} \cup \tau$ is not a strong deformation retract of $B$, then $A^{+}(B) \neq \varnothing$. Isolating blocks enjoy other pleasant properties which enable us to define a homotopy index. This is done in $\$ 4$.

EXAMPLe 2.1. Let $X$ be an open set in $C=C\left([-r, 0], \mathbf{R}^{n}\right), r>0$, and $\pi$ be a local semiflow generated by the solutions of a RFDE

$$
\dot{x}=f\left(x_{t}\right)
$$

where $f: X \rightarrow \mathbf{R}^{n}$ is continuous. Then the closed unit ball $B_{1}$ in $C$ is not an isolating block for $\pi$. In fact, consider a function $\phi \in C$, such that $\left\|\phi\left(\theta_{0}\right)\right\|=1,\|\phi(\theta)\|<1$ for some $\theta_{0} \in(-r, 0)$ and all $\theta \neq \theta_{0}, \theta \in[-r, 0]$. If $x_{t}(\phi)$ is the solution of (2.1) through $\phi$, then $\|x(\phi)(t)\|<1$ for all $0 \leqslant t<\delta, \delta$ a small number. Hence $\left\|x_{t}(\phi)\right\|$ $<1$, and if $t \leqslant \theta_{0}+r$, then $\left\|x_{t}(\phi)\right\|=1$. Hence the solution through $\phi \in \partial B_{1}$ does 
not leave the boundary of $B_{1}$ immediately, and, therefore, $B_{1}$ is not an isolating block.

This situation, which contrasts with the behavior of solutions of ODE'S $(r=0)$, is also related to the following, easily proved assertion:

Let $\dot{x}=L x_{t}$ be an autonomous linear RFDE on $C=C\left([-r, 0], \mathbf{R}^{n}\right), r>0$, and let $S$ be a subspace of $C, \operatorname{dim} S>1$. If there are constants $K, \alpha>0$ such that $\left\|x_{t}(\phi)\right\| \leqslant K e^{-\alpha t}\|\phi\|$ for $\phi \in S$, then necessarily $K>1$.

Again, compare this with ODE's.

EXAMPLE 2.2 (CF. [6]). Consider the $\operatorname{RFDE}\left(2.2_{f}\right)$ :

$$
\dot{x}=L x_{t}+f\left(x_{t}\right)
$$

where $L: C \rightarrow \mathbf{R}^{n}$ is linear and bounded, and $f: \Omega \rightarrow \mathbf{R}^{n}, f \in C^{1}(\Omega), f(0)=0$, $\partial f(0) / \partial x=0$, and

$$
\|f\|_{1}=\sup _{x \in \Omega}\left(\|f(x)\|+\left\|\frac{\partial f}{\partial x}(x)\right\|\right)<\infty .
$$

Suppose that no solution of the characteristic equation $\operatorname{det}\left(\lambda I-L\left(e^{\lambda}\right)\right)=0$ lies on the imaginary axis. Then there are a $\mu>0, \delta>0$ such that whenever $f$ is as before, and $\|f\|_{1}<\mu$, then $\{0\}$ is an isolated invariant set for $\left(2.2_{f}\right)$ and $B_{\delta}=\{\phi \in C \mid\|\phi\|$ $\leqslant \delta\}$ is an isolating neighborhood. Let $T(t): C \rightarrow C$ be the solution operator for $\dot{x}=L x_{t}$. There exists a decomposition $C=U \oplus S, U$ finite-dimensional, such that $T(t) U \subset U, T(t) S \subset S$, on $U, T(t)$ can be uniquely defined for $t \leqslant 0$, and there are constants $K, \alpha>0$ such that

$$
\begin{aligned}
& \|T(t) \phi\| \leqslant K e^{-\alpha t}\|\phi\| \quad \text { for } \phi \in S, t \geqslant 0, \\
& \|T(t) \phi\| \leqslant K e^{+\alpha t}\|\phi\| \quad \text { for } \phi \in U, t \leqslant 0 .
\end{aligned}
$$

Note that if $r>0$, then necessarily $K>1$, by our previous example. If $\Phi$ is any basis for $U$ and $\operatorname{dim} U=d<\infty$, then there is a $d \times d$ matrix $B$ which has only eigenvalues $\lambda$ with re $\lambda>0$ and such that $\dot{y}(t)=B y(t)$ where $y(t)$ is determined by the equation $P_{U}(T(t) \phi)=\Phi \cdot y(t), \phi$ being arbitrary and $P_{U}: C \rightarrow C, P_{S}: C \rightarrow C$ being the projections of $C$ onto $U$ and $S$, respectively. Let $D$ be a positive-definite matrix such that $B^{T} D+D B=I$. ( $B^{T}$ is the transpose of $B$.) Define $V^{+}: C \rightarrow \mathbf{R}$, $V(\phi)=b^{T} D b$, where $P_{U} \phi=\Phi b$. Let

$$
\dot{V}_{f}^{+}(\phi)=\liminf _{t \rightarrow 0^{+}} \frac{1}{t}\left[V^{+}\left(x_{t}(f, \phi)\right)-V^{+}(\phi)\right]
$$

where $x_{t}(f, \phi)$ is the solution of $\left(2.2_{f}\right)$ through $\phi$. It is proved in [6] that there is a $\mu_{1}<\mu$ such that $\dot{V}_{f}^{+}(\phi)>0$, if $\|f\|_{1}<\mu_{1}$ and $\phi \notin S, \phi \in \Omega$. Now choose $\tau>0$ such that $K \cdot e^{-\alpha t}(t+1)<\frac{1}{2}$ for $t \geqslant \tau / 2$. Define $V^{-}: C \rightarrow \mathbf{R}^{+}$

$$
V^{-}(\phi)=\sup _{0<t<\tau}\left[(t+1)\left\|T(t) \cdot P_{S} \phi\right\|\right] \text {. }
$$

Define $\dot{V}_{f}^{-}(\phi)=\lim \sup _{t \rightarrow 0^{+}} t^{-1}\left[V^{-}\left(x_{t}(f, \phi)\right)-V^{-}(\phi)\right]$. It follows from wellknown results of the theory of RFDE's that $V^{-}$is locally Lipschitzian and there are a $0<\delta_{1}<\delta$ and a $0<\mu_{2}<\mu$ such that $\dot{V}_{f}^{-}(\phi)<0$ if $\|f\|_{1}<\mu_{2}$ and $\phi \notin U$, $\phi \in B_{\delta_{1}}$. Now it follows easily that if $\|f\|_{1}<\tilde{\mu}, \tilde{\mu}=\min \left\{\mu_{1}, \mu_{2}\right\}$ and if $\varepsilon$ is sufficiently small, then the set

$$
B=\left\{\phi \in B_{\delta_{1}} \mid V^{+}(\phi) \leqslant \varepsilon, V^{-}(\phi) \leqslant \varepsilon\right\}
$$


is an isolating block for $\left(2.2_{f}\right)$. Obviously Int $B=\left\{\phi \in B_{\delta_{1}} \mid V^{+}(\phi)<\varepsilon, V^{-}(\phi)<\right.$ $\varepsilon\} . B$ and Int $B$ are convex; hence these sets are homeomorphic to the closed (resp. open) unit ball in $C$. Let $B^{+}=\left\{\phi \in \partial B \mid V^{+}(\phi)=\varepsilon\right\}$. Then $B^{+}$is the set of all egress and bounce-off points of $\partial B$ for $\left(2.2_{f}\right)$. Using the mapping $F: B \times[0,1] \rightarrow$ $B, F(\phi, \sigma)=\sigma P_{S} \phi+P_{U} \phi$, it follows that the pair $\left(B, B^{+}\right)$is strongly deformable to $\left(B \cap U, B^{+} \cap U\right)$ and the latter pair is homeomorphic to $\left(K^{d}, S^{d-1}\right)$ where $K^{d}$ is the closed unit sphere in $\mathbf{R}^{d}$, and $S^{d-1}=\partial K^{d}$. This implies in particular that $B^{+}$ is not a retract of Int $B \cup B^{+}$. From the invariance of the homotopy index (defined in \$4) it follows that if $B^{*}$ is any block for $\left(2.2_{f}\right)$ and if $B^{*+}$ is the set of its egress and bounce-off points, then $B^{*+}$ is not a strong deformation retract of $B^{*}$, provided $B^{*+}$ is closed.

REMARKS. For the classical case of two-sided flows on compact spaces, there are several different definitions of an isolating block (cf. [1], [3], [13]). Common to them is the requirement that there should be no internal tangency, i.e., a trajectory coming from the inside of $B$ and reaching a boundary point of $B$ is to leave $B$ immediately. Our definition requires that the trajectory through any boundary point of $B$ leave $B$ immediately in the positive or the negative direction or in both directions. This definition also takes into account that, for semiflows, backward extensions of solutions do not necessarily exist or are unique.

There is no smoothness assumption in our definition which, therefore, resembles the nonsmooth classical case of, e.g., [1]. If $B$ is smooth, i.e. if $\partial B$ is a piecewise $C^{1}$-manifold, or if $B$ is generated by two locally Lipschitzian functions (as in Example 2.2), then $B$ is an isolating block for an open set of flows (in an appropriate topology or a set of flows or semiflows). This makes an isolating block a useful tool in the study of the behavior of a perturbed flow.

If $B$ is not smooth, we cannot, even in the classical case, expect $B$ to be an isolating block for a perturbed flow. However, if the underlying space $X$ is a smooth finite-dimensional manifold and if $\pi$ a smooth (two-sided) flow, then every continuous Liapunov function can be approximated by smooth Liapunov functions, and hence the same is true for isolating blocks generated by Liapunov functions. This fact is proved in [12]. By extending results from [12] to the present, more general case, one should be able to approximate continuous Liapunov functions by locally Lipschitzian functions. This, however, would require substantially new ideas, since the methods used in [12] rely in a very crucial way on the two-sidedness of the flow.

In this paper we shall only study the nonsmooth case. It leads, similarly, to the classical case (cf. [1], [2], [3], [10]) to interesting perturbation results, and, in particular, to the notion of the homotopy index.

Now we shall state a basic existence theorem for isolating blocks:

THEOREM 2.1. If $(X, \pi)$ is a local semiflow on the metric space $X$, and if $(\mathrm{H} 1)-(\mathrm{H} 3)$ hold for the triple $\langle K, U, N\rangle, K \neq \varnothing$, then there exists an isolating block $B$ such that $K \subset$ Int $B \subset B \subset U$.

REMARK. Since the empty set is an isolating block, the theorem is also (trivially) true if $K=\varnothing$. 
The rest of this section is devoted to the proof of Theorem 2.1. To simplify the proof notationally, we shall impose the additional assumption that $\omega_{x}=\infty$ for $x \in X$, i.e. that $\pi$ is a semiflow. The reader may easily adapt the proof to the general case.

The idea of the proof is to construct two Liapunov functions on $U$ and use them to define another isolating neighborhood $\tilde{N}$ of $K$ such that $\tilde{N} \subset U . \tilde{N}$ has nicer properties than $N$, in fact, it is "almost" an isolating block. Now, this process is repeated: analogous Liapunov functions are defined with respect to $\tilde{N}$ rather than $N$. In the same way as before, they are used to define an isolating neighborhood $\tilde{N}$ of $K$ contained in Int $\tilde{N}$. $\tilde{N}$ turns out to satisfy all the properties of an isolating block.

Let us now proceed to the proof: Set

$$
s_{N}^{+}: N \rightarrow \mathbf{R}^{+} \cup\{\infty\}, \quad s_{N}^{+}(x)=\sup \{t \mid x \pi[0, t] \subset N\} .
$$

Define $t_{U}^{+}: U \rightarrow \mathbf{R}^{+} \cup\{\infty\}$ by $t_{U}^{+}(x)=\sup \{t \mid x \pi[0, t] \subset U\}$. Let

$$
\begin{aligned}
F: X \rightarrow[0,1], \quad F(x)=\min \left\{1, \operatorname{dist}\left(x, A^{-}(N)\right)\right\}, \\
G: X \rightarrow[0,1], \quad G(x)=\operatorname{dist}(x, K) /(\operatorname{dist}(x, K)+\operatorname{dist}(x, X \backslash N)) ; \\
g_{U}^{+}(x):=\inf \left\{(1+t)^{-1} G(x \pi t) \mid 0 \leqslant t<t_{U}^{+}(x)\right\}, \\
g_{N}^{-}(x):=\sup \left\{\alpha(t) F(x \pi t) \mid 0 \leqslant t \leqslant s_{N}^{+}(x), \text { if } s_{N}^{+}(x)<\infty,\right. \\
\text { and } \left.0 \leqslant t<\infty, \text { if } s_{N}^{+}(x)=\infty\right\},
\end{aligned}
$$

$g_{U}^{+}$is defined on $U, g_{N}^{-}$is defined on $N, \alpha:[0, \infty) \rightarrow[1,2)$ is a fixed isotone $C^{\infty}$-diffeomorphism.

LEMMA 2.1. (i) $s_{N}^{+}$is upper-semicontinuous, $t_{U}^{+}$is lower-semicontinuous.

(ii) $g_{U}^{+}$is upper-semicontinuous, and $g_{U}^{+}$is continuous in a neighborhood of $K$.

Moreover, if $g_{U}^{+}(x) \neq 0$, then

$$
\dot{g}_{U}^{+}(x)=\liminf _{t \rightarrow 0^{+}}(1 / t)\left(g_{U}^{+}(x \pi t)-g_{U}^{+}(x)\right)>0 .
$$

If $g_{U}^{+}(x)=0$, then for every $t \in \mathbf{R}^{+}, x \pi t \in U$ and $g_{U}^{+}(x \pi t)=0$.

(iii) $g_{N}^{-}$is upper-semicontinuous. If $t_{U}^{+}(x)=s_{N}^{+}(x)$ on $U$, then $g_{N}^{-}$is continuous on $U$.

Moreover, if $g^{-}(x) \neq 0$ then

$$
\dot{g}_{N}^{-}(x)=\limsup _{t \rightarrow 0^{+}}(1 / t)\left(g_{N}^{-}(x \pi t)-g_{N}^{-}(x)\right)<0 .
$$

If $g_{N}^{-}(x)=0$, then for every $t \leqslant s_{N}^{+}(x), g_{N}^{-}(x \pi t)=0$.

Proof of THE LEMMA. To simplify the notation, let us omit the subscripts $U, N$ and simply write $t^{+}, s^{+}, g^{+}, g^{-}$.

(i) is obvious.

(ii) Obviously $g^{+}$is upper-semicontinuous. Let $x_{0}$ be a point of lower semidiscontinuity of $g^{+}$. Then there are $x_{n} \in U, x_{n} \rightarrow x_{0}$, and $\mu>0$ such that $g^{+}\left(x_{0}\right)>\mu$ $>g^{+}\left(x_{n}\right)$. Hence there are $t_{n}, 0 \leqslant t_{n}<t^{+}\left(x_{n}\right)$, for which $\left(1+t_{n}\right)^{-1} G\left(x_{n} \pi t_{n}\right)<\mu<$ $g^{+}\left(x_{0}\right)$. We can assume w.l.o.g. that $t_{n} \rightarrow t_{0} \leqslant \infty$. Obviously $t^{+}\left(x_{0}\right)<\infty$, because otherwise $g^{+}\left(x_{0}\right)=0$. If $t_{0} \leqslant t^{+}\left(x_{0}\right)$, then $\left(1+t_{n}\right)^{-1} G\left(x_{n} \pi t_{n}\right) \rightarrow\left(1+t_{0}\right)^{-1} G\left(x_{0} \pi t_{0}\right)$. 
Hence $\left(1+t_{0}\right)^{-1} G\left(x_{0} \pi t_{0}\right) \leqslant \mu<g^{+}\left(x_{0}\right)$, which is a contradiction. It follows that $t_{0}>t^{+}\left(x_{0}\right)+\delta$ for some $\delta>0$, and we may assume that $t_{n} \geqslant t^{+}\left(x_{0}\right)+\delta$ for all $n$. Suppose there is no neighborhood of $K$ in which $g^{+}$is continuous. Then there exists a sequence $x_{n}$, $\operatorname{dist}\left(x_{n}, K\right) \rightarrow 0$, such that $g^{+}$is not lower-semicontinuous at $x_{n}$. Since $K$ is compact, we may assume that $x_{n} \rightarrow x_{0}, x_{0} \in K$. By what we have just proved, there is a sequence $t_{n}, t^{+}\left(x_{n}\right)<t_{n}<t^{+}\left(y_{n}\right)$, such that dist $\left(y_{n} \pi t^{+}\left(x_{n}\right), \partial U\right)$ $\rightarrow 0$, and $\left(1+t_{n}\right)^{-1} G\left(y_{n} \pi t_{n}\right)<g^{+}\left(x_{n}\right)$. Set $s_{n}=t^{+}\left(x_{n}\right) / 2$. Since $x_{n} \rightarrow x_{0} \in K$ and $K$ is invariant, it follows that $t^{+}\left(x_{n}\right) \rightarrow \infty$. By (H2), the sequence $\left\{x_{n} \pi s_{n}\right\}$ is precompact; hence we may assume w.l.o.g. that $x_{n} \pi s_{n} \rightarrow y_{0} \in A^{-}(N)$. Lemma 1.1 implies that $y_{0} \in A^{+}(N)$. Hence $y_{0} \in A^{-}(N) \cap A^{+}(N)=K$. Hence, $G\left(y_{n} \pi t_{n}\right)<$ $\left(1+t_{n}\right) \cdot\left(1+s_{n}\right)^{-1} \cdot G\left(x_{n} \pi s_{n}\right)$ and $G\left(x_{n} \pi s_{n}\right) \rightarrow G\left(y_{0}\right)=0$. Similarly, it follows that $\left\{y_{n} \pi t^{+}\left(x_{n}\right)\right\}$ is precompact; hence we can assume that $y_{n} \pi t^{+}\left(x_{n}\right) \rightarrow z_{0}, z_{0} \in$ $A^{-}(N) \cap \partial U$.

There are two possible cases:

(1) $\left\{t_{n}-t^{+}\left(x_{n}\right)\right\}$ is bounded; hence w.l.o.g. $t_{n}-t^{+}\left(x_{n}\right) \rightarrow t_{0}<\infty$. Then it follows that $G\left(y_{n} \pi t_{n}\right) \rightarrow 0$, and since for $0 \leqslant s \leqslant t_{n}-t^{+}\left(x_{n}\right),\left(y_{n} \pi t^{+}\left(x_{n}\right)\right) \pi s \in N$, Lemma 1.1 implies that $z_{0} \pi\left[0, t_{0}\right] \subset N$ and $G\left(z_{0} \pi t_{0}\right)=\lim _{n} G\left(y_{n} \pi t_{n}\right)=0$. Hence $z_{0} \pi t_{0} \in K$. This means that $z_{0} \in A^{+}(N) \cap A^{-}(N) \cap \partial U=K \cap \partial U=\varnothing$, a contradiction.

(2) $\left\{t_{n}-t^{+}\left(x_{n}\right)\right\}$ is unbounded; hence w.l.o.g. $t_{n}-t^{+}\left(x_{n}\right) \rightarrow \infty$. It follows that $z_{0} \in A^{+}(N)$, which, again, yields a contradiction.

This proves that $g^{+}$is continuous close to $K$.

Now suppose $g^{+}(x) \neq 0$, i.e. $t^{+}(x)<\infty$, and let $0<s$ be close to 0 . There is a $t_{1}=t_{1}(s)$ such that $s \leqslant t_{1} \leqslant t^{+}(x)$, and $g^{+}(x \pi s)=\left(1+t_{1}-s\right)^{-1} \cdot G\left(x \pi t_{1}\right)$. Hence

$$
\begin{aligned}
(1 / s)\left(g^{+}(x \pi s)-g^{+}(x)\right) & =(1 / s)\left[\left(1+t_{1}-s\right)^{-1} G\left(x \pi t_{1}\right)-g^{+}(x)\right] \\
& \geqslant(1 / s)\left[\left(1+t_{1}-s\right)^{-1}-\left(1+t_{1}\right)^{-1}\right] \cdot G\left(x \pi t_{1}\right) \\
& \geqslant\left(1+t_{1}\right)^{-2} G\left(x \pi t_{1}\right) \geqslant\left(1+t^{+}(x)\right)^{-1} \cdot g^{+}(x) .
\end{aligned}
$$

Hence $\dot{g}^{+}(x)>0$.

The last statement of (ii) is obvious.

(iii) Suppose $g^{-}$is not upper-semicontinuous at $x_{0} \in N$. Hence there are $x_{n} \in N, \mu>0$ such that $x_{n} \rightarrow x_{0}$, and $g^{-}\left(x_{0}\right)<\mu<g^{-}\left(x_{n}\right)$. Consequently there are $t_{n}, t_{n} \leqslant s^{+}\left(x_{n}\right), t_{n}<\infty$, such that $g_{1}^{-}(x)<\mu<\alpha\left(t_{n}\right) \cdot F\left(x_{n} \pi t_{n}\right)$. We can assume $t_{n} \rightarrow t_{0} \leqslant \infty$.

Two cases are possible.

(1) $t_{0}=\infty$. Then, by Lemma 1.1, $\left\{x_{n} \pi t_{n}\right\}$ is precompact and we can assume that $x_{n} \pi t_{n} \rightarrow z_{0} \in A^{-}(N)$. But then $\alpha\left(t_{n}\right) \cdot F\left(x_{n} \pi t_{n}\right) \rightarrow 2 \cdot F\left(z_{0}\right)=0$, which implies $g^{-}(x)<\mu \leqslant 0$, a contradiction.

(2) $t_{0}<\infty$. Then $x_{0} \pi\left[0, t_{0}\right] \subset N$, and $\alpha\left(t_{n}\right) \cdot F\left(x_{n} \pi t_{n}\right) \rightarrow \alpha\left(t_{0}\right) \cdot F\left(x_{0} \pi t_{0}\right)$. This implies on the one hand that $t_{0} \leqslant s^{+}\left(x_{0}\right)$, and on the other hand that $g^{-}\left(x_{0}\right)<\mu<$ $\alpha\left(t_{0}\right) \cdot F\left(x_{0} \pi t_{0}\right) \leqslant g^{-}\left(x_{0}\right)$, a contradiction. We have proved that $g^{-}$is upper-semicontinuous. If $t^{+}=s^{+}$on $U$, then $s^{+}$is continuous on $U$, and this easily implies that $g^{-}$is continuous on $U$. Now let $x \in N$ such that $g^{-}(x) \neq 0$. First assume 
$s^{+}(x)=\infty$. Then, by Lemma 1.1, $x \pi t \rightarrow A^{-}(N)$ and hence $F(x \pi t) \rightarrow 0$, as $t \rightarrow \infty$. For some small $h>0, \inf _{0 \leqslant s \leqslant h} F(x \pi s)=\mu>0$. Let $a>0$ be such that if $t \geqslant a$, then $F(x \pi t)<\mu / 4$. Hence for every $s \leqslant h$, there is a $t_{1}, s \leqslant t_{1} \leqslant a$, such that $g^{-}(x \pi s)=\alpha\left(t_{1}-s\right) \cdot F\left(x \pi t_{1}\right)$. Hence

$$
\begin{aligned}
(1 / s)\left(g^{-}(x \pi s)-g^{-}(x)\right) & \leqslant \frac{1}{s}\left(\alpha\left(t_{1}-s\right)-\alpha\left(t_{1}\right)\right) \cdot \frac{\mu}{2} \\
& =-\frac{1}{s} \cdot \frac{\mu}{2} \int_{t_{1}-s}^{t_{1}} \dot{\alpha}(u) d u \leqslant-\frac{\nu \cdot \mu}{2},
\end{aligned}
$$

where $\nu=\inf _{0 \leqslant u \leqslant a} \dot{\alpha}(u)>0$; hence $\dot{g}^{-}(x) \leqslant-(\nu \cdot \mu) / 2$. The case $s^{+}(x)<\infty$ is dealt with similarly.

The last statement in (iii) is obvious.

LemMa 2.2. Set $H_{\varepsilon}=\left\{x \in U \mid g_{U}^{+}(x)<\varepsilon, g_{N}^{-}(x)<\varepsilon\right\}$. Then $H_{\varepsilon}$ is open, and for $\varepsilon>0$ small enough, it follows that $\mathrm{Cl} H_{\varepsilon} \subset U, g_{U}^{+}$is continuous on $\mathrm{Cl} H_{\varepsilon}$ and $\mathrm{Cl} H_{\varepsilon}$ is an isolating neighborhood of $K$.

Proof of the lemma. We shall drop the subscripts $U, N$ again. Since both $g^{+}$ and $g^{-}$are upper-semicontinuous, $H_{\varepsilon}$ is open for every $\varepsilon>0$. Also, if $x \in K$, then $g^{+}(x)=0=g^{-}(x)$, and hence $K \subset H_{\varepsilon}$. Suppose now that $x_{n} \in U$ are such that $g^{+}\left(x_{n}\right) \rightarrow 0, g^{-}\left(x_{n}\right) \rightarrow 0$. Then $F\left(x_{n}\right) \rightarrow 0$ and, by the compactness of $A^{-}(N)$, there is a subsequence of $\left\{x_{n}\right\}$, denoted by $\left\{x_{n}\right\}$ again, such that $x_{n} \rightarrow x \in A^{-}(N)$.

Now two cases are possible:

(1) $\left\{t^{+}\left(x_{n}\right)\right\}$ is bounded by $B<\infty$. Then $g^{+}\left(x_{n}\right) \geqslant(1+B)^{-1} \inf \left\{G\left(x_{n} \pi t\right) \mid 0 \leqslant t\right.$ $\left.\leqslant t^{+}\left(x_{n}\right)\right\}=(1+B)^{-1} G\left(x_{n} \pi t_{n}\right)$, for some $t_{n}, 0 \leqslant t_{n} \leqslant t^{+}\left(x_{n}\right)$.

We may assume that $t_{n} \rightarrow t_{0}<\infty$. Now Lemma 1.1 implies that $x \pi\left[0, t_{0}\right] \subset N$, and $G\left(x_{n} \pi t_{n}\right) \rightarrow G\left(x \pi t_{0}\right)$. Hence $G\left(x \pi t_{0}\right)=0$, i.e. $x \pi t_{0} \in K$. This implies that $x \in$ $A^{-}(N) \cap A^{+}(N)=K$. Hence $x \in K$.

(2) $\left\{t^{+}\left(x_{n}\right)\right\}$ is unbounded, and we may assume that $t^{+}\left(x_{n}\right) \rightarrow \infty$. Then Lemma 1.1 again implies that $x \in A^{-}(N) \cap A^{+}(N)=K$. We have proved that whenever $g^{+}\left(x_{n}\right) \rightarrow 0$ and $g^{-}\left(x_{n}\right) \rightarrow 0$, then a subsequence of $\left\{x_{n}\right\}$ converges to an element of $K$.

Assume now that $\mathrm{Cl} H_{\varepsilon} \nsubseteq U$ for every $\varepsilon>0$. Then there is a sequence $x_{n}$, $g^{+}\left(x_{n}\right) \rightarrow 0, g^{-}\left(x_{n}\right) \rightarrow 0$, such that $\operatorname{dist}\left(x_{n}, \partial U\right) \rightarrow 0$. By what we have just proved, we can assume that $x_{n} \rightarrow x \in K$. Hence $x \in K \cap \partial U=\varnothing$, a contradiction. Hence, indeed, $\mathrm{Cl} H_{\varepsilon} \subset U$ for $\varepsilon$ small enough.

If there is no $\varepsilon>0$ such that $g^{+}$is continuous on $\mathrm{Cl} H_{e}$, then there are $y_{n} \in \mathrm{Cl} H_{e_{n}}, \varepsilon_{n} \rightarrow 0$, such that $g^{+}$is not continuous at $y_{n}$. Choose $x_{n} \in H_{e_{n}}$, $d\left(x_{n}, y_{n}\right)<\varepsilon_{n}$. Hence $g^{+}\left(x_{n}\right) \rightarrow 0$ and $g^{-}\left(x_{n}\right) \rightarrow 0$, and we may assume that $x_{n} \rightarrow x$ $\in K$. It follows that $y_{n} \rightarrow x \in K$, and this contradicts Lemma 2.1.

The last statement of the lemma is obvious.

We can now complete the proof of Theorem 2.1. Choose $\varepsilon$ so small that $H_{2 e}$ satisfies all properties in Lemma 2.2. Set $\tilde{U}=H_{\varepsilon}, \tilde{N}=\mathrm{Cl} H_{\varepsilon}$. It follows that hypotheses (H1)-(H3) are satisfied for the triple $\langle K, \tilde{U}, \tilde{N}\rangle$. Hence we have the functions $t_{U}^{ \pm}, s_{N}^{ \pm}, g_{U}^{ \pm}, g_{N}^{ \pm}$satisfying Lemma 2.1 with $N, U$ replaced by $\tilde{N}, \tilde{U}$. We 
shall show that $t_{U}^{+}=s_{N}^{+}$on $\tilde{U}$. Indeed, $t_{U}^{+} \leqslant s_{N}^{+}$, of course. Suppose that $t_{U}^{+}(x)<$ $s_{N}^{ \pm}(x)$ for some $x \in \tilde{U}$. It then follows that $y=x \pi t_{U}^{ \pm}(x) \in \partial \tilde{U}$. By Lemma 2.2, $\partial \tilde{U} \subset U$; hence $y \in U$. Hence $g_{U}^{+}(y), g_{N}^{-}(y)$ are defined and either $g_{U}^{+}(y) \geqslant \varepsilon$ or $g_{N}^{-}(y) \geqslant \varepsilon$. If $g_{N}^{-}(y) \geqslant \varepsilon$, then $g_{N}^{-}(x)>0$ and $g_{N}^{-}(y)<g_{N}^{-}(x)$. This implies $g_{N}^{-}(x)$ $>\varepsilon$, which contradicts $x \in \tilde{U}$. Hence $g_{U}^{+}(x) \geqslant \varepsilon$. Now, since $y \in \partial \tilde{U}$, there are $y_{n} \in \tilde{U}$ such that $y_{n} \rightarrow y$. Since $g_{U}^{+}$is continuous on $\mathrm{Cl} H_{2 e}$, it follows that $g_{U}^{+}\left(y_{n}\right) \rightarrow g_{U}^{+}(y)$; hence $g_{U}^{+}(y)=\varepsilon$, i.e. $y \in H_{2 \varepsilon}$. Choose $t$ such that $t_{U}^{ \pm}(x)<t<$ $s_{N}^{+}(x)$. Then $x \pi t \in H_{2 \varepsilon}$. It follows that $g_{U}^{+}(x \pi t)>g_{U}^{+}(y)=\varepsilon$. By continuity of $g_{U}^{+}$ on $\mathrm{Cl}_{2 \varepsilon}$, there is a neighborhood $V$ of $x \pi t$ such that $V \subset H_{2 e}$ and $g_{U}^{+}(z)>\varepsilon$ for $z \in V$. Hence $V \cap H_{\varepsilon}=\varnothing$ and so $x \pi t \notin \mathrm{Cl} H_{\varepsilon}=\tilde{N}$. But this implies $t>s_{\tilde{N}}^{ \pm}(x)$, a contradiction. Hence, indeed, $t_{U}^{+}(x)=s_{N}^{ \pm}(x)$ on $\dot{\tilde{U}}$.

Lemma 2.1 implies that $g_{\tilde{N}}$ is continuous on $\tilde{U}$. Now apply Lemma 2.2 to $\tilde{U}, \tilde{N}$. Hence for all $\delta>0$ sufficiently small, $B_{\delta}=\left\{x \in \tilde{U} \mid g_{U}^{\ddagger}(x)<\delta, g_{\tilde{N}}(x)<\delta\right\}$ has the following properties:

1. $B_{\delta}$ is open, $K \subset B_{\delta}, \mathrm{Cl} B_{\delta} \subset \tilde{U}$.

2. $g_{U}^{\ddagger}$ and $g_{\tilde{N}}$ are continuous on $\mathrm{Cl} B_{\delta}$.

Choose $\delta_{0}=\delta / 2$ and $B=\mathrm{Cl} B_{\delta_{0}}$. We show that $B \subset B_{\delta}$. In fact, $x \in B$ implies $x \in \mathrm{Cl} B_{\delta}$ and there is a sequence $x_{n} \rightarrow x, x_{n} \in B_{\delta_{0}}$. Hence $g_{U}^{ \pm}\left(x_{n}\right)<\delta_{0}, g_{\tilde{N}}\left(x_{n}\right)<$ $\delta_{0}$. Since $g_{U}^{\ddagger}$ and $g_{\tilde{N}}$ are continuous on $\mathrm{Cl} B_{\delta}$, it follows that $g_{U}^{t}(x) \leqslant \delta_{0}<\delta$ and $g_{\bar{N}}(x) \leqslant \delta_{0}<\delta$; hence $x \in B_{\delta}$.

Set

$$
\begin{aligned}
\tilde{b}^{+} & =\left\{x \in \partial B \mid g_{U}^{+}(x)=\delta_{0}, g_{\overline{\tilde{N}}}(x)<\delta_{0}\right\}, \\
\tilde{b}^{-} & =\left\{x \in \partial B \mid g_{U}^{+}(x)<\delta_{0}, g_{\tilde{N}}(x)=\delta_{0}\right\}, \\
\tilde{\tau} & =\left\{x \in \partial B \mid g_{U}^{ \pm}(x)=\delta_{0}, g_{\tilde{\tilde{N}}}(x)=\delta_{0}\right\} .
\end{aligned}
$$

It follows that $\partial B=\tilde{b}^{+} \cup \tilde{b}^{-} \cup \tilde{\tau}$.

Using the properties of $g_{U}^{ \pm}$and $g_{\tilde{N}}$ it is easily proved that every point of $\tilde{b}^{+}$is a strict egress point, every point of $\tilde{b}^{-}$is a strict ingress point and every point of $\tilde{\tau}$ is a bounce-off point. Hence $B$ is an isolating block, and this completes the proof of Theorem 2.1.

3. Index pairs. As was pointed out in the previous section, a (nonsmooth) isolating block, in general, is not stable under perturbations of the semiflow. Although, at first, this seems to be an annoying drawback of this concept, it turns out that, similar to the classical case of nonsmooth blocks for flows on compact spaces (cf. [1] and [10]), there is an important feature of an isolating block that does not change under small perturbations of the semiflow, namely, the homotopy type of the quotient space $B /\left(b^{+} \cup \tau\right)$. While proving this fact in $\S \S 3$ and 4, we develop the theory of index pairs for semiflows satisfying (H2). We borrow many ideas from C. C. Conley's excellent monograph [2], in which the theory of index pairs is developed for semiflows on locally compact spaces. However, our proofs are usually quite different from those in [2], since we do not assume local compactness of the underlying space. 
We shall see that the method of Liapunov functions, used in the previous section, can also be applied to prove the existence and several properties of index pairs. In this way Liapunov functions become a unifying technique in the construction of both isolating blocks and index pairs.

In this section, we shall define index pairs, prove their existence and study their behavior under perturbations of the semiflow.

Definition 3.1 (CF. [2]). Let $\pi$ be a local semiflow on the metric space $X$. Let $K$ be an isolated invariant set for $\pi$, and let $N$ be an isolating neighborhood of $K$.

An ordered pair of sets $\left\langle N_{1}, N_{2}\right\rangle$ is called an index pair in $N$ if:

(1) $N_{1}, N_{2}$ are closed subsets of $N$, and $N_{1}, N_{2}$ are $N$-positively invariant.

(2) $K \subset \operatorname{Int}\left(N_{1} \backslash N_{2}\right)$.

(3) If $x \in N_{1}$ and $t>0, t<\omega_{x}, x \pi t \notin N$, then there exists a $t^{\prime}<t$ such that $x \pi\left[0, t^{\prime}\right] \subset N_{1}$ and $x \pi t^{\prime} \in N_{2}$.

RemarK. A subset $A \subset N$ is called $N$-positively invariant, if $x \in A$ and $x \pi[0, t]$ $\subset N$ imply $x \pi[0, t] \subset A$.

EXAMPLE 3.1. If $B$ is an isolating block for $K$, then $\left\langle B, B^{+}\right\rangle$, where $B^{+}=b^{+} \cup$ $\tau$, is an index pair in $N=B$, provided $B^{+}$is closed. Note that $B^{+}$is closed for the isolating block $B$ constructed in the proof of Theorem 2.1 .

EXAMPLE 3.2. Let $X=C([-r, 0], \mathbf{R})$ and $\pi$ be generated by the RFDE

$$
\dot{x}=a x(t)+b x(t-r), \quad a>|b| \text {. }
$$

Then $K=\{0\}$ is an isolated invariant set for $\pi$ and $N=B_{1}=\{\phi|| \phi \mid \leqslant 1\}$ is an isolating neighborhood for $K$. Set $N_{1}=N, N_{2}=\{\phi \in N|| \phi(0) \mid=1\}$. Then $\left\langle N_{1}, N_{2}\right\rangle$ is an index pair in $N$.

ReMARK. If $\left\langle N_{1}, N_{2}\right\rangle$ is an index pair in $N$, then so is $\left\langle N_{1}, N_{1} \cap N_{2}\right\rangle$. Hence it can always be assumed that $N_{2} \subset N_{1}$.

We shall now prove a basic existence theorem for index pairs.

THEOREM 3.1. Let (H1)-(H3) be satisfied not only for the triple $\langle K, U, N\rangle$ but also for $\langle K, \tilde{U}, \tilde{N}\rangle$, where $\tilde{U}$ is open, $\tilde{N}=\mathrm{Cl} \tilde{U}$, and $N \subset \tilde{U}$. Let $\tilde{W}$ be open, $K \subset \tilde{W}$. Then there exists an index pair $\left\langle N_{1}, N_{2}\right\rangle$ in $N$ such that $\mathrm{Cl}\left(N_{1} \backslash N_{2}\right) \subset \tilde{W}$.

Proof. If $K=\varnothing$, let $N_{1}=N=N_{2}$. Then $\left\langle N_{1}, N_{2}\right\rangle$ obviously is an index pair in $N$.

Suppose therefore that $K \neq \varnothing$. Let $V$ be an open set such that $K \subset V, \mathrm{Cl} V \subset$ $U \cap \tilde{W}$. Since (H1)-(H3) are satisfied for $\langle K, \tilde{U}, \tilde{N}\rangle$, the functions $g_{U}^{ \pm}, g_{\tilde{N}}$ are defined as in the proof of Theorem 2.1. Choose $\varepsilon>0$ in such a way that $\mathrm{Cl}\left\{x \in \tilde{U} \mid g_{\tilde{N}}(x)<\varepsilon\right.$ and $\left.g_{U}^{+}(x)<\varepsilon\right\} \subset V$. This is possible by the proof of Lemma 2.2.

Define

$$
N_{1}=N \cap \mathrm{Cl}\left\{x \in \tilde{U} \mid g_{\tilde{N}}(x)<\varepsilon\right\}, \quad N_{2}=N \backslash\left\{x \in \tilde{U} \mid g_{U}^{\ddagger}(x)<\varepsilon\right\} .
$$

We shall show that $\left\langle N_{1}, N_{2}\right\rangle$ is an index pair in $N$.

(1) $N_{1}, N_{2} \subset N$ and $N_{1}$ is closed, and since $g_{U}^{t}$ is upper-semicontinuous, $N_{2}$ is closed. 
Let $y \in N_{1}, y \pi[0, s] \subset N$. Then there are $x_{n} \in \tilde{U}, g_{\tilde{N}}\left(x_{n}\right)<\varepsilon, x_{n} \rightarrow y$. For $n$ large enough, $x_{n} \pi[0, s]$ is defined and $\subset \tilde{U}$. For every $\tilde{s}, 0 \leqslant \tilde{s} \leqslant s$, it follows that $x_{n} \pi \tilde{S} \rightarrow y \pi \tilde{s}$. Since $g_{\tilde{N}}\left(x_{n} \pi \tilde{S}\right) \leqslant g_{\tilde{N}}\left(x_{n}\right)<\varepsilon$, it follows that $y \pi \tilde{s} \in N_{1}$. Hence $N_{1}$ is $N$-positively invariant.

Now let $y \in N_{2}, y \pi[0, s] \subset N$. Then for $0 \leqslant \tilde{s} \leqslant s, y \pi \tilde{s} \in \tilde{U}$; hence $g_{U}^{+}(y \pi s)$ is defined. Since $g_{U}^{ \pm}(y \pi s)>g_{U}^{\ddagger}(y) \geqslant \varepsilon$, it follows that $y \pi \tilde{s} \in N_{2}$. Consequently $N_{2}$ is $N$-positively invariant.

(2) Obviously $K \subset W:=\left\{x \in \tilde{U} \mid g_{\tilde{\tilde{N}}}(x)<\varepsilon, g_{U}^{ \pm}(x)<\varepsilon\right\} . W$ is open, $\mathrm{Cl} W \subset$ $V$, and $W \subset N_{1} \backslash N_{2}$.

(3) Let $x \in N_{1}, t<\omega_{x}, x \pi t \notin N_{1}$. Let $t^{\prime}=s_{N}^{+}(x)$. Then $t^{\prime}<t<\infty$, and $x \pi\left[0, t^{\prime}\right]$ $\subset N$.

By (1), $x \pi\left[0, t^{\prime}\right] \subset N_{1}$. Suppose that $x \pi t^{\prime} \notin N_{2}$. Then $g_{U}^{ \pm}\left(x \pi t^{\prime}\right)<\varepsilon$. This implies that $x \pi t^{\prime} \in \mathrm{Cl} W \subset V$. But this yields a contradiction to the definition of $t^{\prime}$.

(4) It remains to be shown that $\mathrm{Cl}\left(N_{1} \backslash N_{2}\right) \subset \tilde{W}$. Indeed, $N_{1} \backslash N_{2} \subset V \subset \mathrm{Cl} V$ $\subset U \cap \tilde{W}$. The proof is complete.

In order to formulate the perturbation theorem, we have to consider more than one local semiflow. The following concepts turn out to be expedient.

Definition 3.2. Let $\left\{\pi_{n}\right\}$ be a sequence of local semiflows on $X$, and let $\pi$ be a local semiflow on $X$. The pair $\left\langle\left\{\pi_{n}\right\}, \pi\right\rangle$ is said to satisfy the continuity property, if for every choice of $x_{n}, x \in X$, and $t_{n}, t \in \mathbf{R}^{+}$, for which $x_{n} \rightarrow x, t_{n} \rightarrow t, t<\omega_{x}$, it follows that $t_{n}<\omega_{x_{n}}$ for all $n$ sufficiently large, and $x_{n} \pi t_{n} \rightarrow x \pi t$.

If $N$ is a closed subset of $X$, then $\left\langle\left\{\pi_{n}\right\}, \pi\right\rangle$ is called $N$-admissible if for every choice of $x_{n} \in X$ and $t_{n} \in \mathbf{R}^{+}$for which $x_{n} \pi_{n}\left[0, t_{n}\right] \subset N$ and $t_{n} \rightarrow \infty$, it follows that the sequence $\left\{x_{n} \pi_{n} t_{n}\right\}$ is precompact.

Now the following lemma holds:

Lemma 3.1. Suppose $\left\langle\left\{\pi_{n}\right\}, \pi\right\rangle$ satisfies the continuity property and is $N$-admissible. Suppose that $N$ and $\pi$ satisfy (H3).

(a) If $N$ is an isolating neighborhood with respect to $\pi$, then $N$ is also an isolating neighborhood with respect to all $\pi_{n}, n$ sufficiently large.

(b) Every cluster point of a sequence $\left\{x_{n} \pi_{n} t_{n}\right\}, x_{n} \pi_{n}\left[0, t_{n}\right] \subset N, t_{n} \rightarrow \infty$, is an element of $A_{\pi}^{-}(N)$.

The easy proof of the lemma is omitted.

Let $\Phi$ be a set of local semiflows on $X$. Let $\sigma$ be a topology on $\Phi$ which satisfies the first axiom of countability. We shall say that $\mathcal{T}$ satisfies the continuity property (or that $\mathcal{T}$ is $N$-admissible, resp.) if, for every sequence $\pi_{n} \in \Phi$ such that $\pi_{n} \rightarrow \pi$ in $\mathcal{T}$, the pair $\left\langle\left\{\pi_{n}\right\}, \pi\right\rangle$ satisfies the continuity property (or is $N$-admissible, resp.).

EXAMPLE 1.1 (CONTINUED). Let $D$ be a fixed stable difference operator on $C$. Let $\mathscr{F}(\Omega)$ be the set of all bounded continuous mappings $f: \Omega \rightarrow \mathbf{R}^{n}$ having the property that the associated NFDE $d D\left(x_{t}\right) / d t=f\left(x_{t}\right)$ has a unique solution through every $\phi \in \Omega$. Let $\mathscr{F}(\Omega)$ be given the sup-norm topology: $\|f\|=\sup _{x \in \Omega}\|f(x)\|$. Let $\Phi$ be the set of all local semiflows on $X=\Omega$ generated by mappings in $\mathscr{F}(\Omega)$. The mapping $\mathscr{F}(\Omega) \ni f \rightarrow \pi_{f} \in \Phi$ associating with every $f \in \mathscr{F}(\Omega)$ the corresponding local semiflow is bijective. Let us endow $\Phi$ with the uniquely determined topology 
$\mathcal{T}$ for which the mapping $f \rightarrow \pi_{f}$ is a homeomorphism. $\mathcal{T}$ is obviously metrizable; hence it satisfies the first axiom of countability. Using a slight modification of the argument proving (H2) in Example 1.1, one can show that $\mathscr{T}$ is $N$-admissible for every closed and bounded subset $N$ of $X$. Also, it follows from basic results on NFDE's (cf. [6]) that $\mathscr{T}$ satisfies the continuity property.

EXAMPLE 1.2 (CONTINUED). Let $A$ be a fixed sectorial operator, $0 \leqslant \alpha<1$ be arbitrary, $U \subset X^{\alpha}$ be open. Denote by $\mathcal{L}$ the set of all bounded, locally Lipschitzian mappings $f: U \rightarrow X$ endowed with the sup-norm topology. Let $\beta$ be the mapping, associating with every $\mu \in(0, \infty)$ and every $f \in \mathcal{L}$ the local semiflow on $U$ generated by the solutions of the equation

$$
d u / d t+\mu \cdot A u=f(u), \quad u(0)=u_{0} .
$$

Assume that the resolvent $R(\lambda ; A)$ is compact for every $\lambda \in \rho(A)$. Then $\beta$ is injective. Let $\Phi$ be the image of $(0, \infty) \times \mathcal{L}$ under $\beta$. Give $\Phi$ the uniquely determined topology $\mathcal{T}$ for which $\beta$ is a homeomorphism. Obviously, $\mathcal{T}$ is metrizable. $\mathcal{T}$ satisfies the continuity property, this being proved in [7]. Also, it can be proved by using results in [7] that $\mathscr{T}$ is $N$-admissible for every closed bounded set $N$ in $U$.

Lemma 3.2. Let $V, U, \tilde{U}$ be open sets, $N=\mathrm{Cl} U, \tilde{N}=\mathrm{Cl} \tilde{U}, V \subset \mathrm{Cl} V \subset U \subset N$ $\subset \tilde{U} \subset \tilde{N}$. Suppose that the largest $\pi$-invariant set $K_{\pi}\left(K_{\pi}\right.$ may be empty $)$ in $\tilde{N}$ is included in $V$. Define $t_{\pi}^{+}$with respect to $\tilde{U}$ and $\pi$ as in the proof of Theorem 2.1, i.e. $t_{\pi}^{+}(x)=\{t \geqslant 0 \mid x \pi[0, t]$ is defined and $\subset \tilde{U}\}$, for $x \in \tilde{U}$.

Then for every $M>0$ the sets

$$
\begin{aligned}
& N_{1, \pi}=N \cap \mathrm{Cl}\{y \mid \text { there are } x \in V, t \geqslant 0, \text { such that } x \pi[0, t] \subset \tilde{U} \text { and } x \pi t=y\}, \\
& N_{2, \pi}=N_{1, \pi} \cap\left\{y \in \tilde{U} \mid t_{\pi}^{+}(y) \leqslant M\right\},
\end{aligned}
$$

enjoy the following properties:

(1) $N_{1, \pi}$ and $N_{2, \pi}$ are closed and $N$-positively invariant subsets of $N$,

(2) $K_{\pi} \subset V \cap\left\{y \in \tilde{U} \mid t_{\pi}^{+}(Y)>M\right\} \subset N_{1, \pi} \backslash N_{2, \pi}$,

(3) $V \subset N_{1, \pi}$

Proof. (2) and (3) are obvious, (1) is an easy exercise.

LEMMA 3.3. Let $\left\langle\left\{\pi_{n}\right\}, \pi\right\rangle$ be a pair satisfying the continuity property and suppose $\tilde{N}$ is a $\pi$-isolating block for $K_{\pi}, K_{n} \neq \varnothing$. Let $\tilde{U}=\operatorname{Int} \tilde{N}$. Assume that $\left\langle\left\{\pi_{n}\right\}, \pi\right\rangle$ is $\tilde{N}$-admissible and that $\tilde{\pi}$ and $\tilde{N}$ satisfy $(\mathrm{H} 2)$ and $(\mathrm{H} 3)$ (with $\pi$ and $N$ replaced by $\tilde{\pi}$ and $\tilde{N})$, where $\tilde{\pi}$ stands for $\pi$ and for every $\pi_{n}$. If $\pi^{\prime}$ is a local semiflow on $X$ and $x \in \tilde{U}$, set

$$
t_{\pi^{\prime}}^{+}(x)=\sup \left\{t \geqslant 0 \mid x \pi^{\prime}[0, t] \text { is defined and } \subset \tilde{U}\right\} .
$$

Moreover, for $x \in \tilde{N}$ define $g^{-}:=g_{\bar{N}}$ as in the proof of Theorem 2.1, with $N$ replaced by $\tilde{N}$. Let $a, b, M_{0}>0$ be constants, $M_{0}>b$, and define

$$
U=\left\{x \in \tilde{U} \mid g^{-}(x)<a, t_{\pi}^{+}(x)>b\right\}, \quad N=\mathrm{Cl} U \text {. }
$$


Choose $a, b$ so that $N \subset \tilde{U}$. Under these assumptions, there exist an $\varepsilon_{0}>0$ such that for all $\varepsilon \leqslant \varepsilon_{0}$ there is an $n_{0}=n_{0}\left(\varepsilon, M_{0}\right)$ such that for all $n \geqslant n_{0}$ properties (1) and (2) below hold:

(1) The largest $\pi_{n}$-invariant set $K_{\pi_{n}}$ in $\tilde{N}$ is included in $V=V\left(\varepsilon, M_{0}\right)=\{x \in$ $\left.\tilde{U} \mid g^{-}(x)<\varepsilon, t_{\pi}^{+}(x)>M_{0}\right\}$.

(2) If $N_{1, \pi_{n}}$ and $N_{2, \pi_{n}}$ are defined as in Lemma 3.2 with respect to $V=V\left(\varepsilon, M_{0}\right)$ (we shall write $N_{1, \pi_{n}}=N_{1, \pi_{n}}\left(\varepsilon, M_{0}\right)$ and $N_{2, \pi_{n}}=N_{2, \pi_{n}}\left(\varepsilon, M_{0}\right)$ ), then $\left\langle N_{1, \pi_{n}}, N_{2, \pi_{n}}\right\rangle$ is an index pair in $N$.

Proof. Suppose the lemma is not true. In view of Lemmas 3.1 and 3.2 we can then assume w.l.o.g. that there are $\varepsilon_{n}>0, y_{n}$ such that $\varepsilon_{n} \rightarrow 0, y_{n} \in N_{1, \pi_{n}}\left(\varepsilon_{n}, M_{0}\right) \cap$ $\partial N, y_{n} \notin N_{2, \pi_{n}}\left(\varepsilon_{n}, M_{0}\right)$. It follows that $t_{\pi_{n}}^{+}\left(y_{n}\right)>M_{0}$. By the definition of $N_{1, \pi_{n}}$, there are $y_{k, n}, x_{k, n}, t_{k, n} \geqslant 0$ such that $\lim _{k \rightarrow \infty} y_{k, n}=y_{n}, x_{k, n} \in V\left(\varepsilon_{n}, M_{0}\right), x_{k, n} \pi_{n}\left[0, t_{k, n}\right] \subset$ $\tilde{U}, x_{k, n} \pi_{n} t_{k, n}=y_{k, n}$. There is a subsequence $k_{n} \rightarrow \infty$ such that $d\left(y_{k_{n}, n}, y_{n}\right) \rightarrow_{n} 0$, $x_{k_{n}, n} \rightarrow_{n} x_{0} \in A^{-}(\tilde{N})$.

Let $y_{n}^{\prime}=y_{k_{n}, n}, x_{n}^{\prime}=x_{k_{n}, n}, t_{n}^{\prime}=t_{k_{n}, n}$. It follows that $x_{n}^{\prime} \pi_{n}\left[0, t_{n}^{\prime}\right] \subset \tilde{U}, x_{n}^{\prime} \pi_{n}\left(t_{n}^{\prime}\right) \rightarrow \partial N$. Now the $\tilde{N}$-admissibility and the continuity property of $\left\langle\left\{\pi_{n}\right\}, \pi\right\rangle$ implies that $\left\{x_{n}^{\prime} \pi_{n}\left(t_{n}^{\prime}\right)\right\}$ is precompact, and we may assume that $x_{n}^{\prime} \pi_{n}\left(t_{n}^{\prime}\right) \rightarrow y_{0}, y_{0} \in A_{\pi}^{-}(\tilde{N}) \cap$ $\partial N$. Since $t_{\pi_{n}}^{+}\left(y_{n}\right)>M_{0}$, it follows from the fact that $\tilde{N}$ is an isolating block that $t_{\pi}^{+}\left(y_{0}\right) \geqslant M_{0}$; hence $y_{0} \in U \cap \partial N=\varnothing$, a contradiction. Hence the lemma is proved.

LemMA 3.4. Let all assumptions of Lemma 3.3 be satisfied. Then for every $M>0$ there is an $\varepsilon_{0}^{\prime}=\varepsilon_{0}^{\prime}(M)$ and an $n_{0}^{\prime}=n_{0}^{\prime}(M)$ such that, for every $\varepsilon \leqslant \varepsilon_{0}^{\prime}$ and every $n \geqslant n_{0}^{\prime}, \quad\left\{x \in \tilde{U} \mid t_{\pi_{\alpha}}^{+}(x) \leqslant 2 M\right\} \cap \mathrm{Cl} V(\varepsilon, M) \subset\left\{x \in \tilde{U} \mid t_{\pi}^{+}(x) \leqslant 3 M\right\} \cap$ $\mathrm{Cl} V(\varepsilon, M) \subset\left\{x \in \tilde{U} \mid t_{\pi_{n}}^{+}(x) \leqslant 4 M\right\} \cap N_{1, \pi_{n}}(\varepsilon, M) \subset\left\{x \in \tilde{U} \mid t_{\pi}^{+}(x)<5 M\right\} \cap N$.

The proof of Lemma 3.4 is left to the reader, who may supply it by using ideas from the proof of Lemma 3.3 .

Combining Lemmas 3.2, 3.3 and 3.4 we arrive at the following perturbation theorem for index pairs.

THEOREM 3.2. Let $\left\langle\left\{\pi_{n}\right\}, \pi\right\rangle$ be a pair satisfying the continuity property. Suppose $\tilde{N}$ is a $\pi$-isolating neighborhood of $K_{\pi}, K_{\pi} \neq \varnothing$. Assume that $\left\langle\left\{\pi_{n}\right\}, \pi\right\rangle$ is $\tilde{N}$-admissible and that $\tilde{\pi}$ and $\tilde{N}$ satisfy $(\mathrm{H} 2)$ and $(\mathrm{H} 3)$ (with $\pi$ and $N$ replaced by $\tilde{\pi}$ and $\tilde{N})$, where $\tilde{\pi}$ stands for $\pi$ and for every $\pi_{n}$. Then there exist two $\pi$-isolating neighborhoods $N^{\prime}$ and $N$ and two index pairs $\left\langle N_{1, \pi}^{\prime}, N_{2, \pi}^{\prime}\right\rangle$ and $\left\langle N_{1, \pi}, N_{2, \pi}\right\rangle$ for $\pi$ in $N^{\prime}$ and $N$, resp., such that for all $\pi_{n}$, $n$ sufficiently large, the following properties hold:

(1) $K_{\pi} \subset N^{\prime} \subset$ Int $N \subset$ Int $\tilde{N}, N_{1, \pi}^{\prime}=N^{\prime}, N_{1, \pi}=N$.

(2) The largest $\pi_{n}$-invariant set $K_{\pi_{n}}$ in $\tilde{N}$ is included in $N^{\prime}$.

(3) There are two index pairs $\left\langle N_{1, \pi_{n}}^{\prime}, N_{2, \pi_{n}}^{\prime}\right\rangle$ and $\left\langle N_{1, \pi_{n}}, N_{2, \pi_{n}}\right\rangle$ for $\pi_{n}$ in $N^{\prime}$ and $N$, resp., such that $N_{1, \pi_{n}}^{\prime} \subset N_{1, \pi}^{\prime} \subset N_{1, \pi_{n}} \subset N_{1, \pi}, N_{2, \pi_{n}}^{\prime} \subset N_{2, \pi}^{\prime} \subset N_{2, \pi_{n}} \subset N_{2, \pi}$.

4. The homotopy index. In this section, we shall consider the set $\mathcal{S}$ of pairs $(\pi, K)$, where $\pi \in \Phi$ and $K$ is an isolated, $\pi$-invariant set. We want to discuss perturbations of $(\pi, K)$, and, to this end, similarly as in the classical case, we introduce a topology on $\mathcal{S}$, making $\mathcal{S}$ a sheaf over $\Phi$. With this topology we shall 
complete the construction of the homotopy index, by proving its independence of a particular choice of an isolating neighborhood $N$ or an index pair in $N$, and by showing its homotopy invariance. Now a slight, although important, extension of the continuity property for the Alexander-Spanier cohomology theory is used to prove that $N_{1} / N_{2}$ and $N_{1} \cap A^{-}(N) / N_{2} \cap A^{-}(N)$ generate the same AlexanderSpanier cohomology modules. In particular, under certain additional assumptions, $A^{-}(N)$ has finite topological dimension. In this case, $N_{1} / N_{2}$ cohomologically behaves like a finite-dimensional set. Heuristically, this latter result can serve as a theoretical justification of the use of Ważewski's principle and certain methods from algebraic topology to such infinite-dimensional problems as the qualitative analysis of RFDE's, NFDE's or PDE's.

Before proceeding to the results of this section, let us recall a few facts from homotopy theory.

A pair $(X, A)$, where $X$ is a topological space and $A$ is a subspace, is called a topological pair. A pointed space is a pair $(X, p)$, where $X$ is a topological space and $p \in X$. If $(X, A)$ and $(Y, B)$ are topological pairs, then a morphism $g:(X, A) \rightarrow$ $(Y, B)$ is a continuous mapping $g: X \rightarrow Y$ such that $g[A] \subset B$. Similarly, if $(X, p)$ and $(Y, q)$ are pointed spaces, then a morphism $g:(X, p) \rightarrow(Y, q)$ is a continuous mapping $g: X \rightarrow Y$ such that $g(p)=q$.

Let $(X, A)$ be a topological pair. If $A \neq \varnothing$, call two points $x, y$ equivalent, if either $x=y$ and $x \notin A$, or if both $x$ and $y$ are in $A$. This clearly defines an equivalence relation on $X$. Denote by $X / A$ the pointed space $(X / \sim, p)$, where $X / \sim$ is the quotient space induced by this equivalence relation, and $p$ is the common equivalence class of all elements of $A . X / \sim$ is endowed with the quotient topology.

If $A=\varnothing$, then choose any point $p \notin X$, give $\{p\}$ the discrete topology, let $X \cup\{p\}$ have the sum topology, and define $X / A=X / \varnothing=X \cup\{p\} /\{p\}$. Since different choices of $p$ yield isomorphic spaces $X \cup\{p\} /\{p\}$, there is no need to distinguish between them. Abusing the notation, we shall frequently write $X / A$ for $X / \sim$ (or $X \cup\{p\} / \sim$, if $A=\varnothing$ ), but no confusion should arise. Also, if $x \in X$ (or $x \in X \cup\{p\}$, resp.), then $[x]$ is the equivalence class of $x$.

If $g:(X, A) \rightarrow(Y, B)$ is a morphism, then there exists a uniquely determined morphism $\hat{g}: X / A \rightarrow Y / B$ such that the following diagram is commutative:

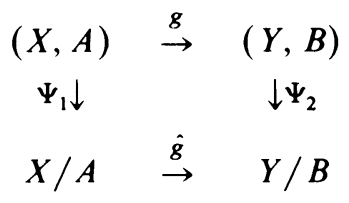

Here, $\Psi_{1}$ (resp. $\Psi_{2}$ ) sends every element of $X$ (resp. of $Y$ ) to its equivalence class in $X / A$ (resp. in $Y / B)$.

$\hat{g}$ is called the morphism induced by $g$.

If $(X, p)$ is a pointed space, then define $(X, p) \times[0,1]=(X \times[0,1],\{p\} \times$ $[0,1])$. If $f, g:(X, p) \rightarrow(Y, q)$ are two morphisms, then by a homotopy from $f$ to $g$ we mean a morphism $F:(X, p) \times[0,1] \rightarrow(Y, q)$ such that $F(x, 0)=f(x)$ and 
$F(x, 1)=g(x)$ for all $x \in X$. If there is a homotopy from $f$ to $g$, we write $f \sim g$. This defines an equivalence relation on the set of all morphisms from $(X, p)$ to $(Y, q)$.

We say that $f:(X, p) \rightarrow(Y, q)$ is a homotopy equivalence, if there is a morphism $g:(Y, q) \rightarrow(X, p)$ such that $f \circ g \sim 1(Y, q)$ and $g \circ f \sim 1(X, p)$, where $1(Y, q)$ and $1(X, p)$ are the identities in $(Y, q)$ and $(X, p)$, respectively.

If there is a homotopy equivalence from $(X, p)$ to $(Y, q)$, then we say that $(X, p)$ and $(Y, q)$ are of the same homotopy type, and write $(X, p) \sim(Y, q)$.

If we use the Kelley-Morse axiomatization of the set theory (see [16]), then $\sim$ is an equivalence relation on the class $\mathscr{Z}$ of all pointed spaces ( $\mathscr{Z}$ is not a set). Let $[X, p]$ be the equivalence class of $(X, p)$. Then $[X, p]$ is called the homotopy type of $(X, p)$.

If the following diagram

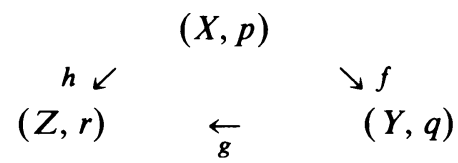

commutes and any two of the morphisms are homotopy equivalences, then so is the third morphism.

Let us finally note that if $X$ is a normal space and if $A$ and $Y$ are closed subets of $X$, then the map $i: Y / Y \cap A \rightarrow Y \cup A / A$ induced by the inclusion $(Y, Y \cap A)$ $\rightarrow(Y \cup A, A)$ is an isomorphism.

As in $\$ 3$, let $\Phi$ be a set of local semiflows on $X$, endowed with a first-countable topology $\mathcal{T}$ satisfying the continuity property. Let $\mathcal{N}$ be the class of all closed sets $N$ such that (H3) holds for every $\pi \in \Phi$ and $\mathcal{T}$ is $N$-admissible. In particular, (H2) holds for $N$ and every $\pi \in \Phi$. Now let $\delta$ be the set of all pairs $(\pi, K)$, where $\pi \in \Phi$, such that there exists an $N \in \mathcal{R}$ with the property that $K$ is an isolated $\pi$-invariant set and $N$ is an isolating neighborhood of $K$. We shall endow $\mathcal{S}$ with a "natural" topology, generated in the following way: Let $N \in \mathcal{R}$. By Lemma 3.1 the set $\Phi(N)=\{\pi \in \Phi \mid N$ is an isolating neighborhood with respect to $\pi\}$ is open in $\Phi$. Define $\gamma[N]: \Phi(N) \rightarrow \mathcal{S}, \pi \rightarrow(\pi, \mathfrak{s}(\pi, N))$, where $\mathfrak{s}(\pi, N)$ is the largest $\pi$-invariant set in $N$. Since $\pi \in \Phi(N), \mathfrak{S}(\pi, N) \subset$ Int $N$; hence $\mathfrak{S}(\pi, N)$ is an isolated invariant set and it follows that $(\pi, \mathfrak{g}(\pi, N)) \in \mathcal{S}$. Hence $\gamma[N]$ is well defined.

Now the following lemma holds, which is analogous to Lemma 6.3 in [10]:

LEMMA 4.1. The set $\{\operatorname{Im}(\gamma[N] \mid V) \mid N \in \mathfrak{N}, V$ open in $\Phi\}$ is a basis for a topology on $\mathcal{S}$; i.e. if $N_{1}, N_{2} \in \mathcal{N}, V_{1}, V_{2}$ are open in $\Phi$, and $(\pi, K) \in \operatorname{Im}\left(\gamma\left[N_{1}\right] \mid V_{1}\right) \cap$ $\operatorname{Im}\left(\gamma\left[N_{2}\right] \mid V_{2}\right)$, then there are $N \in \mathfrak{N}$ and $V$ open in $\Phi$ such that

$$
(\pi, K) \in \operatorname{Im}(\gamma[N] \mid V) \subset \operatorname{Im}\left(\gamma\left[N_{1}\right] \mid V_{1}\right) \cap \operatorname{Im}\left(\gamma\left[N_{2}\right] \mid V_{2}\right) .
$$

Proof. Let $N=N_{1} \cap N_{2}$. Then $N \in \Re$. We show that there is an open neighborhood $V^{\prime}$ of $\pi$ such that $\mathfrak{S}\left(\pi^{\prime}, N\right)=\mathfrak{S}\left(\pi^{\prime}, N_{1}\right)=\mathfrak{I}\left(\pi^{\prime}, N_{2}\right)$ for $\pi^{\prime} \in V$. Suppose this is not true. Then w.l.o.g. we can assume that there are $\pi_{n} \in \Phi, \pi_{n} \rightarrow \pi$, and $x_{n} \in \mathfrak{S}\left(\pi_{n}, N_{1}\right) \backslash N$. There are $y_{n}$ and $t_{n}$, such that $y_{n} \pi_{n}\left[0, t_{n}\right] \subset N_{1}, t_{n} \rightarrow \infty$, and 
$y_{n} \pi_{n} t_{n}=x_{n}$. The $N_{1}$-admissibility of $\mathcal{T}$ implies that $\left\{x_{n}\right\}$ is precompact and we may assume w.l.o.g. that $x_{n} \rightarrow x \in A_{\pi}^{-}\left(N_{1}\right)$. Obviously $x \in A_{\pi}^{+}\left(N_{1}\right)$. Hence $x \in$ $\mathfrak{S}(\pi, M)$. Hence $x \in \operatorname{Int} N$, which is a contradiction to our choice of $x_{n}$. Let $V=V^{\prime} \cap V_{1} \cap V_{2}$. Then $\Phi(N) \cap V \subset \Phi\left(N_{1}\right) \cap \Phi\left(N_{2}\right)$. This obviously implies the lemma.

With the topology of $S$ defined by Lemma $4.1, \gamma[N]$ becomes a homeomorphism of $\Phi(N)$ onto its range. Define $p: \delta \rightarrow \Phi$ by projection: $p(\pi, K)=\pi$. Then $p$ is locally a homeomorphism, i.e. for every $(\pi, K)$ there is a neighborhood $W$ of $(\pi, K)$ such that $p \mid W$ is a homeomorphism of $W$ onto $p[W]$. In other words, $\delta$ is a sheaf over $\Phi$. As in the classical case, $\delta$ is a $T_{1}$-space, if $\Phi$ is a $T_{1}$-space, but $\delta$ is not necessarily $T_{2}$, even if $\Phi$ is metrizable.

We shall say that two elements $(\pi, K)$ and $\left(\pi^{\prime}, K^{\prime}\right)$ of $\mathcal{S}$ are related by continuation, if $(\pi, K),\left(\pi^{\prime}, K^{\prime}\right)$ both belong to the same quasicomponent of $\delta$ (i.e. to a common connected subset of $\delta$ ). This obviously defines an equivalence relation on $\delta$. For instance, if $\pi$ and $\pi^{\prime}$ both belong to a common connected subset of $\Phi(N)$, then $(\pi, \mathfrak{s}(\pi, N))$ and $\left(\pi^{\prime}, \mathfrak{s}\left(\pi^{\prime}, N\right)\right)$ are related by continuation. This latter statement is, of course, much more significant if we also know that $\Phi$ is locally connected. This is certainly the case in Example 1.2 (PDE), and it is also true in Example 1.1 if instead of $\Phi$ we consider the subspace $\Phi^{\prime}$ of $\Phi$ containing all local semiflows generated by $d D\left(x_{t}\right) / d t=f\left(x_{t}\right)$, with $f$ locally Lipchitizian.

We shall prove in this section that for any isolated $\pi$-invariant set $K$ and any two index pairs $\left(N_{1}, N_{2}\right)$ (in $N$ ), and $\left(\bar{N}_{1}, \bar{N}_{2}\right)$ (in $\bar{N}$ ), where $N, \bar{N}$ are two isolating neighborhoods of $K$, it turns out that $N_{1} / N_{1} \cap N_{2}$ and $\bar{N}_{1} / \bar{N}_{1} \cap \bar{N}_{2}$ are of the same homotopy type. Hence we can define an object $i(\pi, K)$, called the homotopy index of $(\pi, K)$, and which is equal to the homotopy type of any index pair $\left(N_{1}, N_{2}\right)$ for $K$. The second result states that $i(\pi, K)$ is an invariant of continuation, i.e. if $(\pi, K)$ and $\left(\pi^{\prime}, K^{\prime}\right)$ are related by continuation, then $i(\pi, K)=i\left(\pi^{\prime}, K^{\prime}\right)$. We shall now study in greater detail several homotopy equivalences induced by the local semiflow $\pi$ on $X$.

Let $N$ be an isolating neighborhood for $K$ (with respect to a local semiflow $\pi$ ), satisfying (H3). Let $\left\langle N_{1}, N_{2}\right\rangle$ be an index pair in $N$.

Set for $t \geqslant 0$ :

$N_{1}^{t}=\left\{x \mid\right.$ there is a $y \in N_{1}$ such that $y \pi[0, t] \subset N$ and $\left.y \pi t=x\right\}$,

$N_{2}^{-t}=\left\{x \mid\right.$ there is a $0 \leqslant t^{\prime} \leqslant t$, such that $x \pi\left[0, t^{\prime}\right] \subset N$ and $\left.x \pi t^{\prime} \in N_{2}\right\}$.

$N_{1}^{t}$ and $N_{2}^{-t}$ are $N$-positively invariant,

$N_{1}^{t} \subset N_{1}, N_{2}^{-t} \supset N_{2}$, and $N_{2}^{-t}$ is closed.

$N_{1}^{t}$ is not closed, in general.

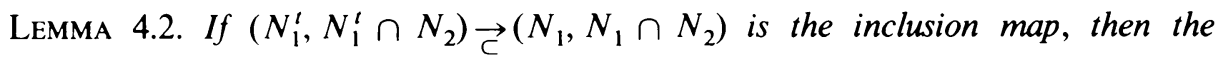
induced map $i: N_{1}^{t} / N_{1}^{t} \cap N_{2} \rightarrow N_{1} / N_{1} \cap N_{2}$ is a homotopy equivalence.

Proof. Let $\hat{p}, \hat{q}$ be the distinguished points of $N_{1}^{t} / N_{1} \cap N_{2}$ and $N_{1} / N_{1} \cap N_{2}$, resp. Define $f: N_{1} / N_{1} \cap N_{2} \rightarrow N_{1}^{t} / N_{1}^{t} \cap N_{2}$ by

$$
f(z)= \begin{cases}{[x \pi t]} & \text { if }[x]=z, x \pi[0, t] \subset N_{1}, \\ \hat{p} & \text { otherwise; }\end{cases}
$$




$$
\begin{gathered}
F: N_{1} / N_{1} \cap N_{2} \times[0,1] \rightarrow N_{1} / N_{1} \cap N_{2}, \\
F(z, \sigma)= \begin{cases}{[x \pi \sigma t]} & \text { if }[x]=z, x \pi[0, \sigma t] \subset N_{1}, \\
\hat{q} & \text { otherwise; }\end{cases} \\
G: N_{1}^{t} / N_{1}^{t} \cap N_{2} \times[0,1] \rightarrow N_{1}^{t} / N_{1}^{t} \cap N_{2}, \\
G(z)= \begin{cases}{[x \pi \sigma t]} & \text { if }[x]=z, x \pi[0, \sigma t] \subset N_{1}, \\
\hat{p} & \text { otherwise. }\end{cases}
\end{gathered}
$$

Properties of $N_{1}, N_{2}$ imply that $f, F$, and $G$ are well defined. Suppose we know that all these maps are continuous. It is easily checked that $i \circ f=F_{1}, f \circ i=G_{1}$. But $F$ yields a homotopy between $F_{1}$ and $1\left(N_{1} / N_{1} \cap N_{2}\right)$, and $G$ yields a homotopy between $G_{1}$ and $1\left(N_{1}^{t} / N_{1}^{t} \cap N_{2}\right)$. Hence $i$ is, in fact, a homotopy equivalence.

It remains to be shown that $f, F$ and $G$ are continuous. The proofs being similar in all three cases, we shall only prove that $f$ is continuous, if $N_{1}^{t} \cap N_{2} \neq \varnothing$. All the remaining cases are left to the reader. Let $z=[x] \in N_{1} / N_{1} \cap N_{2}$ and let $U$ be an open set in $N_{1}^{t} / N_{1}^{t} \cap N_{2}$ for which $f(z) \in U$. There is an open set $\tilde{W}$ in $X$ such that $U=\left\{[y]\right.$ in $\left.N_{1}^{t} / N_{1}^{t} \cap N_{2} \mid y \in \tilde{W} \cap N_{1}^{t}\right\}$.

Two cases are possible:

$1^{\circ}$ case. $x \pi[0, t] \subset N_{1} \backslash N_{2}$. Then there is an open set $\tilde{V}$ in $x$ such that $x \in \tilde{V}$ and $\tilde{V} \pi[0, t] \subset X \backslash N_{2}$, and $\tilde{V} \pi t \in \tilde{W}$. Since points in $N_{1}$ cannot leave $N_{1}$ without hitting $N_{2}$, it follows that $\left(\tilde{V} \cap N_{1}\right) \pi t \in \tilde{W} \cap N_{1}^{t}$. Let $V=\left\{[y]\right.$ in $N_{1} / N_{1} \cap N_{2} \mid y$ $\left.\in \tilde{V} \cap N_{1}\right\}$. Then $V$ is open in $N_{1} / N_{1} \cap N_{2}, z=[x] \in V$, and $f[V] \subset U$.

$2^{\circ}$ case. $x \pi[0, t] \nsubseteq N_{1} \backslash N_{2}$. Then $f(z)=\hat{p}=\left[N_{1}^{t} \cap N_{2}\right]$. Hence $N_{1}^{t} \cap N_{2} \subset \tilde{W}$. Let $\tilde{V}=\left\{y \in X \mid y \pi[0, t] \nsubseteq N_{1}\right.$ or $\left.y \pi t \in \tilde{W}\right\}$. Then $x \in \tilde{V}$ and $\tilde{V}$ is open in $x$. Also, $N_{1} \cap N_{2} \subset \tilde{V}$. Let $V=\left\{[y]\right.$ in $\left.N_{1} / N_{1} \cap N_{2} \mid y \in \tilde{V} \cap N_{1}\right\} . V$ is open in $N_{1} / N_{1} \cap N_{2},[x] \in V$ and it is easily proved that $f[V] \subset U$.

Hence $f$ is, indeed, continuous.

LEMMA 4.3. If $\left(N_{1}, N_{1} \cap N_{2}\right) \rightarrow\left(N_{1}, N_{1} \cap N_{2}^{-t}\right)$ is the inclusion map, then the induced map $j: N_{1} / N_{1} \cap N_{2} \rightarrow N_{1} / N_{1} \cap N_{2}^{-t}$ is a homotopy equivalence.

Proof. Let $\hat{q}$ and $\hat{r}$ be the distinguished points of $N_{1} / N_{1} \cap N_{2}$ and $N_{1} / N_{1} \cap$ $N_{2}^{-t}$, resp. Let $g: N_{1} / N_{1} \cap N_{2}^{-t} \rightarrow N_{1} / N_{1} \cap N_{2}$ be defined as

$$
g(z)=\left\{\begin{array}{ll}
{[x \pi t]} & \text { if }[x]=z, x \pi[0, t] \subset N_{1} \backslash N_{2}, \\
\hat{q} & \text { otherwise. }
\end{array} .\right.
$$

Define $H: N_{1} / N_{1} \cap N_{2}^{-t} \times[0,1] \rightarrow N_{1} / N_{1} \cap N_{2}^{-t}$ by

$$
H(z, \sigma)= \begin{cases}{[x \pi \sigma t]} & \text { if }[x]=z, x \pi[0, \sigma t] \subset N_{1} \backslash N_{2}, \\ \hat{r} & \text { otherwise. }\end{cases}
$$

Similarly to the preceding lemma, it follows that $g, H$ are well defined and continuous. 
Now $g \circ j=F_{1}, F$ being defined in the proof of Lemma 4.2. Hence

$$
g \circ j \sim 1\left(N_{1} / N_{1} \cap N_{2}\right), \quad j \circ g=H_{1},
$$

and $H$ gives a homotopy from $H_{1}$ to $1\left(N_{1} / N_{1} \cap N_{2}^{-1}\right)$.

The lemma is proved.

Let $\bar{N}, N$ be two isolating neighborhoods for $K$, and assume that $K \neq \varnothing$. Suppose furthermore that $\bar{N} \subset N$, (H2), (H3) are satisfied for $N$, and that $\left(\bar{N}_{1}, \bar{N}_{2}\right)$, $\left(N_{1}, N_{2}\right)$ are two index pairs in $\bar{N}, N$ resp., such that $\left(\bar{N}_{1}, \bar{N}_{2}\right) \subset\left(N_{1}, N_{2}\right)$. Then it is easily proved that there is a $t>0$ such that:

(1) whenever $x \in N_{1}$ and $x \pi[0, t] \subset N_{1} \backslash N_{2}^{-t}$ then $x \pi t \in \bar{N}_{1}$;

(2) if $x \in \bar{N}_{1} \cap N_{2}$ then $x \pi[0, t] \nsubseteq \bar{N}_{1}$.

With this choice of $t$, the following lemma holds:

LEMMA 4.4. The inclusion induced maps $i_{1}: \bar{N}_{1} / \bar{N}_{1} \cap N_{2}^{-t} \rightarrow N_{1} / N_{1} \cap N_{2}^{-t}$, and $j_{1}: \bar{N}_{1} / \bar{N}_{1} \cap \bar{N}_{2} \rightarrow \bar{N}_{1} / \bar{N}_{1} \cap N_{2}$ are homotopy equivalences.

The proof of Lemma 4.4 uses only ideas employed in the preceding lemmas, and it is left to the reader.

Corollary. Suppose $\bar{N} \subset N,\left(\bar{N}_{1}, \bar{N}_{2}\right) \subset\left(N_{1}, N_{2}\right),(\mathrm{H} 2)-(\mathrm{H} 3)$ holds for $N, \bar{N}$ and $N$ are isolating neighborhoods of $K$, and $\left(\bar{N}_{1}, \bar{N}_{2}\right),\left(N_{1}, N_{2}\right)$ are index pairs in $\bar{N}$ and $N$, respectively.

Then the inclusion induced map $e: \bar{N}_{1} / \bar{N}_{1} \cap \bar{N}_{2} \rightarrow N_{1} / N_{1} \cap N_{2}$ is a homotopy equivalence.

Proof. Suppose first that $K \neq \varnothing$. Consider the sequence of inclusion induced maps:

$$
\bar{N}_{1} / \bar{N}_{1} \cap \bar{N}_{2} \underset{j_{1}}{\rightarrow} \bar{N}_{1} / \bar{N}_{1} \cap N_{2} \underset{j_{2}}{\rightarrow} \bar{N}_{1} / \bar{N}_{1} \cap N_{2}^{-t} \underset{i_{1}}{\rightarrow} N_{1} / N_{1} \cap N_{2}^{-t},
$$

where $t$ is as in Lemma 4.4. Lemmas 4.3 and 4.4 imply that $i_{1} \circ j_{2} \circ j_{1}$ is a homotopy equivalence. But $i_{1} \circ j_{2} \circ j_{1}=i_{2} \circ e$, where $i_{2}$ is the inclusion induced map $i_{2}: N_{1} / N_{1} \cap N_{2} \rightarrow N_{1} / N_{1} \cap N_{2}^{-t}$. Since also $i_{2}$ is a homotopy equivalence, it follows that $e$ is a homotopy equivalence.

Suppose now that $K=\varnothing$. Then $\left(\bar{N}_{1}, \bar{N}_{1}\right)$ and $\left(N_{1}, N_{1}\right)$ are index pairs in $\bar{N}$ and $N$, resp. Consider the sequence of maps:

$$
\bar{N}_{1} / \bar{N}_{1} \underset{f}{\rightarrow} \bar{N}_{1} / \bar{N}_{1} \cap \bar{N}_{2} \underset{e}{\rightarrow} N_{1} / N_{1} \cap N_{2} \rightarrow \underset{g}{\rightarrow} N_{1} / N_{1}
$$

where $\bar{f}$ is the unique morphism of the pointed one-point space into $\bar{N}_{1} / \bar{N}_{1} \cap \bar{N}_{2}$, and $g$ is inclusion induced.

Since $g \circ e \circ \bar{f}$ is an isomorphism, the lemma will be proved if we can show that $g$ and $\bar{f}$ are homotopy equivalences. Of course, it is enough to show that the unique map $f: N_{1} / N_{1} \rightarrow N_{1} / N_{1} \cap N_{2}$ is a homotopy inverse of $g$.

Obviously $g \circ f=1\left(N_{1} / N_{1}\right)$. Let $F: N_{1} / N_{1} \cap N_{2} \times[0,1] \rightarrow N_{1} / N_{1} \cap N_{2}$ be defined as in Lemma 4.2. Since $K=\varnothing$, there exists a $t>0$ such that if $x \in N_{1}$, then $x \pi[0, t] \oplus N_{1}$. With this choice of $t, F(z, 1)=\hat{p}$, where $\hat{p}$ is the distinguished point of $N_{1} / N_{1} \cap N_{2}$. Hence $F_{1}=f \circ g$, and it follows that

$$
f \circ g \sim 1\left(N_{1} / N_{1} \cap N_{2}\right) .
$$


The corollary is proved.

LEMMA 4.5. Let $N$ be an isolating neighborhood for $K, K \neq \varnothing$, and let $\left(N_{1}, N_{2}\right)$ and $\left(\bar{N}_{1}, \bar{N}_{2}\right)$ be two index pairs in $N$. Suppose $(\mathrm{H} 2)$ and $(\mathrm{H} 3)$ hold for $N$. Then there exists a $t \in \mathbf{R}^{+}$such that $N_{1}^{t} \subset \bar{N}_{1}$ and $N_{1}^{t} \cap N_{2} \subset \bar{N}_{1} \cap \bar{N}_{2}^{-t}$.

Proof. There is a $t_{0}$ such that whenever $y \pi\left[0,2 t_{0}\right] \subset N$, then $y \pi t_{0} \in \operatorname{Int}\left(\bar{N}_{1} \backslash \bar{N}_{2}\right)$. Let $t \geqslant 2 t_{0}$, and $x \in N_{1}^{t}$ be arbitrary. Then $y \pi\left[0,2 t_{0}\right] \subset N_{1} \subset N$ and $y \pi 2 t_{0}=x$. Hence $y \pi t_{0} \in \bar{N}_{1}$ and by $N$-positive invariance of $\bar{N}_{1}$ it follows that $x \in \bar{N}_{1}$. Now assume that $\bar{N}_{1} \cap N_{2} \nsubseteq \bar{N}_{2}^{-t}$ for every $t \in \mathbf{R}^{+}$. Then there are $x_{n} \in \bar{N}_{1} \cap N_{2}$, $t_{n} \rightarrow \infty$, such that $x_{n} \pi\left[0, t_{n}\right] \cap \bar{N}_{2}=\varnothing$. It follows that $x_{n} \pi\left[0, t_{n}\right] \subset \bar{N}_{1} \subset N$ and hence $x_{n} \pi\left[0, t_{n}\right] \subset N_{2}$. Let $y_{n}=x_{n} \pi\left(t_{n} / 2\right)$. Then $y_{n}$ can be assumed to converge to $y_{0} \in K$. But then $y_{0} \in K \cap N_{2}$, a contradiction.

The lemma is proved.

LEMMA 4.6. Let $N$ be a $\pi$-isolating neighborhood, satisfying (H3).

Then the inclusion induced map e: $N_{1}^{t} / N_{1}^{t} \cap N_{2} \rightarrow \mathrm{Cl} N_{1}^{t} / \mathrm{Cl} N_{1}^{t} \cap N_{2}$ is a homotopy equivalence.

Proof. Define $f: \mathrm{Cl} N_{1}^{t} / \mathrm{Cl} N_{1}^{t} \cap N_{2} \rightarrow N_{1}^{t} / N_{1}^{t} \cap N_{2}$ by

$$
f(z)= \begin{cases}{[x \pi t],} & \text { if }[x]=z \text { and } x \pi[0, t] \subset N_{1} \backslash N_{2}, \\ \hat{p}, & \text { otherwise. }\end{cases}
$$

Set $F: \mathrm{Cl} N_{1}^{t} / \mathrm{Cl} N_{1}^{t} \cap N_{2} \times[0,1] \rightarrow \mathrm{Cl} N_{1}^{t} / \mathrm{Cl} N_{1}^{t} \cap N_{2}$ to be

$$
F(z, \sigma)= \begin{cases}{[x \pi \sigma t],} & \text { if }[x]=z \text { and } x \pi[0, \sigma t] \subset N_{1} \backslash N_{2}, \\ \hat{q}, & \text { otherwise. }\end{cases}
$$

Finally, let $G$ be defined as in the proof of Lemma 4.2.

It follows from the properties of an index pair that $F$ and $f$ are well defined. Now the proof is completed similarly to that of Lemma 4.2.

We are now ready to state and prove the main theorems of this section:

TheOREM 4.1. Let $(\pi, K) \in \mathcal{S}$ and suppose $N, \bar{N} \in \Re$ are two isolating neighborhoods of $K$.

If $\left(N_{1}, N_{2}\right)$ and $\left(\bar{N}_{1}, \bar{N}_{2}\right)$ are index pairs in $N$ and $\bar{N}$, respectively, then $N_{1} / N_{1} \cap$ $N_{2}$ and $\bar{N}_{1} / \bar{N}_{1} \cap \bar{N}_{2}$ are of the same homotopy type.

Proof. Suppose first that $K=\varnothing$. Then $\left(N_{1}, N_{1}\right)$ is an index pair in $N$. By the corollary of Lemma 4.4, $N_{1} / N_{1} \cap N_{2}$ has the same homotopy type as $N_{1} / N_{1}$. But $N_{1} / N_{2}$ is a pointed one-point space. Hence the result.

Now assume that $K \neq \varnothing$.

$1^{\circ}$ case. $N=\bar{N}$; by Lemma 4.5 , there is a $t \geqslant 0$ such that $\left(N_{1}^{t}, N_{1}^{t} \cap N_{2}\right) \subset$ $\left(\bar{N}_{1}, \bar{N}_{1} \cap \bar{N}_{2}^{-t}\right)$ and $\left(\bar{N}_{1}^{t}, \bar{N}_{1}^{t} \cap \bar{N}_{2}\right) \subset\left(N_{1}, N_{1} \cap N_{2}^{-t}\right)$. Let $g: N_{1} / N_{1} \cap N_{2}^{-t} \rightarrow$ $N_{1}^{t} / N_{1}^{t} \cap N_{2}$ and $\bar{g}^{\prime}: \bar{N}_{1} / \bar{N}_{1} \cap \bar{N}_{2}^{-t} \rightarrow \bar{N}_{1}^{t} / \bar{N}_{1}^{t} \cap \bar{N}_{2}$ be defined as

$$
g^{\prime}(z)= \begin{cases}{[x \pi t]} & \text { if }[x]=z, x \pi[0, t] \subset N_{1} \backslash N_{2}, \\ \hat{p} & \text { otherwise. }\end{cases}
$$

Similarly for $\bar{g}^{\prime}$. Here, $\hat{p}$ is the distinguished point of $N_{1}^{t} / N_{1} \cap N_{2}$. It follows from earlier results in this section that $g^{\prime}$ and $\bar{g}^{\prime}$ are homotopy equivalences. 
Consider the sequence of maps:

$$
\begin{gathered}
N_{1}^{t} / N_{1}^{t} \cap N_{2} \stackrel{i_{1}}{\rightarrow} \bar{N}_{1} / \bar{N}_{1} \cap \bar{N}_{2}^{-t} \stackrel{\bar{g}^{\prime}}{\rightarrow} \bar{N}_{1}^{t} / \bar{N}_{1}^{t} \cap \bar{N}_{2} \stackrel{i_{2}}{\rightarrow} N_{1} / N_{1} \cap N_{2}^{-t} \\
\stackrel{g^{\prime}}{\rightarrow} N_{1}^{t} / N_{1}^{t} \cap N_{2} \stackrel{i_{1}}{\rightarrow} \bar{N}_{1} / \bar{N}_{1} \cap \bar{N}_{2}^{-t}
\end{gathered}
$$

where $i_{1}$ and $i_{2}$ are inclusion induced.

Now $i_{2} \circ \bar{g}^{\prime} \circ i_{1}=i_{2}^{\prime} \circ g \circ i_{1}^{\prime}$, where $i_{1}^{\prime}: N_{1}^{t} / N_{1}^{t} \cap N_{2} \rightarrow N_{1} / N_{1} \cap N_{2}^{-t}$ and $i_{2}^{\prime}$ : $N_{1} / N_{1} \cap N_{2} \rightarrow N_{1} / N_{1} \cap N_{2}^{-t}$ are inclusion induced, and $g$ is defined in the proof of Lemma 4.3. Hence $i_{2} \circ \bar{g}^{\prime} \circ i_{1}$ is a homotopy equivalence. By symmetry, $i_{1} \circ g^{\prime} \circ i_{2}$ is also a homotopy equivalence.

Hence it follows that $i_{1}$ and $i_{2}$ are homotopy equivalences. It follows, therefore, that $N_{1}^{t} / N_{1}^{t} \cap N_{2}$ and $\bar{N}_{1} / \bar{N}_{1} \cap \bar{N}_{2}^{-t}$ are of the same homotopy type, which, by Lemmas 4.2 and 4.3 , implies that $N_{1} / N_{1} \cap N_{2}$ and $\bar{N}_{1} / \bar{N}_{1} \cap \bar{N}_{2}$ are of the same homotopy type.

$2^{\circ}$ case. $N \neq \bar{N}$.

By $1^{\circ}$ case, it is enough to prove the assertion of the lemma for some specially chosen index pairs $\left(N_{1}, N_{2}\right)$ and $\left(\bar{N}_{1}, \bar{N}_{2}\right)$.

Choose $\left(\bar{N}_{1}, \bar{N}_{2}\right)$ and $\left(N_{1}, N_{2}\right)$ in such a way that $\operatorname{Cl}\left(N_{1} \backslash N_{2}\right) \subset N \cap \bar{N}$, $\mathrm{Cl}\left(\bar{N}_{1} \backslash \bar{N}_{2}\right) \subset N \cap \bar{N}, N_{2} \subset N_{1}, \bar{N}_{2} \subset \bar{N}_{1}$. This is possible by Theorem 3.1. Then $\left(N_{1} \cap \bar{N}, N_{2} \cap \bar{N}\right)$ and $\left(\bar{N}_{1} \cap N, \bar{N}_{2} \cap N\right)$ are both index pairs in $N \cap \bar{N}$. Now, by the corollary of Lemma 4.4, $N_{1} \cap \bar{N} / N_{2} \cap \bar{N}$ and $N_{1} / N_{2}$ have the same homotopy type. Similarly for $\bar{N}_{1} \cap N / \bar{N}_{2} \cap N$ and $\bar{N}_{1} / \bar{N}_{2}$. By what we have shown before $N_{1} \cap \bar{N} / N^{2} \cap \bar{N}$ and $\bar{N}_{1} \cap N / \bar{N}^{2} \cap N$ are of the same homotopy type. It follows that $N_{1} / N_{2}$ has the same homotopy type as $\bar{N}_{1} / \bar{N}_{2}$.

The theorem is proved.

As was mentioned earlier, Theorem 4.1 permits us to define an object $i(\pi, K)$, called the homotopy index of $(\pi, K)$ and equal to the homotopy type of an arbitrary index pair $\left(N_{1}, N_{2}\right)$ in $N$ (with respect to $\pi$ ), where $N$ is an arbitrary $\pi$-isolating neighborhood of $K$ satisfying (H2) and (H3).

We shall now show that $i(\pi, K)$ is a homotopy invariant.

THEOREM 4.2. If $(\pi, K)$ and $\left(\pi^{\prime}, K^{\prime}\right)$ are related by continuation, then $i(\pi, K)=$ $i\left(\pi^{\prime}, K^{\prime}\right)$.

Proof. It suffices to show that the set of all $(\pi, K)$ having the same homotopy type is open. Let $\left(\pi_{0}, K_{0}\right)$ be arbitrary. Let $N \in \mathcal{N}$ be an isolating neighborhood for $K$. Suppose first that $K_{0}=\varnothing$. Hence $\mathfrak{s}\left(\pi_{0}, N\right)=\varnothing$. Hence, by Lemma 3.1, there is a neighborhood $V$ of $\pi_{0}$ in $\Phi$ such that $\mathfrak{s}\left(\pi_{0}, N\right)=\varnothing$ for $\pi \in V$. The set $\tilde{U}=\operatorname{im}(\gamma[N] \cap V)$ is open in $\delta,\left(\pi_{0}, K_{0}\right) \in \tilde{U}$, and for every $(\pi, K) \in \tilde{U},(N, N)$ is an index pair for $\pi$ in $N$. Hence $i(\pi, K)$ is equal to the homotopy type of $N / N$, a pointed one-point space. The theorem follows for $K_{0}=\varnothing$.

Suppose now that $K_{0} \neq \varnothing$. Apply Theorem 3.1, and consider the sequence of inclusion induced maps

$$
N_{1, \pi}^{\prime} / N_{1, \pi}^{\prime} \cap N_{2, \pi}^{\prime} \stackrel{i_{1}}{\rightarrow} N_{1, \pi_{0}}^{\prime} / N_{1, \pi_{0}}^{\prime} \cap N_{2, \pi_{0}}^{\prime} \stackrel{i_{2}}{\rightarrow} N_{1, \pi} / N_{1, \pi} \cap N_{2, \pi} \stackrel{i_{3}}{\rightarrow} N_{1, \pi_{0}} / N_{1, \pi_{0}} \cap N_{2, \pi_{0}}
$$


By the corollary of Lemma 4.4 it follows that $i_{2} \circ i_{1}$ and $i_{3} \circ i_{2}$ are homotopy equivalences. Hence $i_{1}, i_{2}, i_{3}$ are homotopy equivalences, from which the theorem follows immediately.

THEOREM 4.3. Let $\tilde{\Phi}$ be the set of all local semiflows on $X$ (no topology is imposed on $\tilde{\Phi})$. Let $E$ be a first countable topological space, and let $\varepsilon_{0}$ be a point of $E$. Suppose a mapping $\pi: E \rightarrow \tilde{\Phi}$ and a closed set $N$ are given such that the following properties are satisfied:

(a) For every $\varepsilon \in E, \pi(\varepsilon)$ satisfies $(\mathrm{H} 3)$ and $(\mathrm{H} 2)$ for $N$.

(b) $N$ is an isolating neighborhood for $\pi\left(\varepsilon_{0}\right)$.

(c) Whenever $\varepsilon_{n} \rightarrow \varepsilon_{0}$, then the pair $\left\langle\left\{\pi\left(\varepsilon_{n}\right)\right\}, \pi\right\rangle$ is $N$-admissible and satisfies the continuity property.

Under these assumptions, there is a neighborhood $U$ of $\varepsilon_{0}$ in $E$ such that, for every $\varepsilon \in U, N$ is an isolating neighborhood for $\pi(\varepsilon)$ and $i(\pi(\varepsilon), \mathfrak{s}(\pi(\varepsilon), N))=$ $i\left(\pi\left(\varepsilon_{0}\right), \mathfrak{S}\left(\pi\left(\varepsilon_{0}\right), N\right)\right)$.

The proof of Theorem 4.3 is very similar to that of Theorem 4.2 and is, therefore, omitted.

Corollary. Let $\tilde{\Phi}, E, \pi$, and $N$ be as in Theorem 4.3. Suppose that $E$ is connected and that (a), (b) and (c) of Theorem 4.3 hold for every $\varepsilon_{0} \in E$.

Then for every $\varepsilon, \varepsilon^{\prime} \in E$

$$
i(\pi(\varepsilon), \mathfrak{S}(\pi(\varepsilon), N))=i\left(\pi\left(\varepsilon^{\prime}\right), \mathfrak{S}\left(\pi\left(\varepsilon^{\prime}\right), N\right)\right) .
$$

REMARKS. There is a formal similarity between the homotopy index and the fixed point index for local-condensing maps (see [4] and [15]). This follows from our basic hypothesis $(\mathrm{H} 2)$, from the definition of an isolating neighborhood, from Theorem 4.1 ("excision property"), and from Theorems 4.2 and 4.3, especially the corollary to Theorem 4.3 ("homotopy invariance"). Also, note the following:

Let $\overline{0}$ be the homotopy type of a pointed one-point space. Then $i(\pi, \varnothing)=\overline{0}$. Hence, if $i(\pi, K) \neq \overline{0}$, then $K \neq \varnothing$.

EXAMPLE 2.2 (CONTINUED). If $K=\{0\}$ is the isolated invariant set generated by the local semiflow in Example 2.1 (a hyperbolic equilibrium) then $i(\pi, K)=\Sigma^{d}$, where $\Sigma^{d}$ is the homotopy type of the pointed $d$-sphere $S^{d}$ (in $\mathbf{R}^{d+1}$ ).

EXAMPle 4.1. Let $\alpha_{0}>0, F:\left(-\alpha_{0}, \alpha_{0}\right) \times C \rightarrow \mathbf{R}^{n}$. Suppose that, for every $\alpha$, $F(\alpha, \cdot)$ is a completely continuous, locally Lipschitzian mapping. Let $\pi(\alpha)$ be the local semiflow on $C$ generated by the RFDE $\dot{x}=F\left(\alpha, x_{t}\right)$. Suppose that $F(\alpha, 0)=$ $0, \alpha \in\left(-\alpha_{0}, \alpha_{0}\right)$ and that $K_{\alpha}=\{0\}$ is an isolated invariant set for every $\alpha$. Also, if $\alpha \neq 0$, then assume that $K_{\alpha}=\{0\}$ is a hyperbolic equilibrium point of $\dot{x}=$ $F\left(\alpha, x_{t}\right)$, and that whenever $\alpha>0$ and $\alpha^{\prime}<0$, then the dimensions of the corresponding unstable manifolds $U_{\alpha}$ and $U_{\alpha^{\prime}}$ of $K_{\alpha}$ and $K_{\alpha^{\prime}}$, resp., are different.

Finally, assume that there is a closed, bounded neighborhood $N_{0} \subset C$ of 0 , such that whenever $\alpha_{n} \rightarrow 0$, then the pair $\left\langle\left\{\pi\left(\alpha_{n}\right)\right\}, \pi\right\rangle$ is $N_{0}$-admissible and satisfies the continuity property. (This assumption is e.g. satisfied, if $F(\alpha, \phi)$ is differentiable with respect to $\alpha$ and $\sup _{|\alpha| \leqslant \alpha_{1} ; \phi \in N_{0}}\left\|F_{\alpha}^{\prime}(\alpha, \phi)\right\|<\infty$ for some $\alpha_{1} \leqslant \alpha_{0}$ and some closed neighborhood $N_{0}$ of 0 .) 
Under these assumptions, for every neighborhood $N$ of 0 in $C$ and every $\alpha_{2}>0$ there are an $\alpha \in\left(-\alpha_{0}, \alpha_{0}\right),|\alpha| \leqslant \alpha_{2}$, and a solution $x_{t}^{\alpha} \neq 0$ of $\dot{x}(t)=F\left(\alpha, x_{t}\right)$ defined for all $t \in(-\infty, \infty)$ and such that $x_{t}^{\alpha} \in N$ for all $t \in \mathbf{R}$.

Indeed, otherwise there exist a $\pi(0)$-isolating neighborhood $N \subset N_{0}$ of $K_{0}$ and an $\alpha_{2}$, such that $N$ is a $\pi(\alpha)$-isolating neighborhood for $|\alpha| \leqslant \alpha_{2}$ and $B(\pi(\alpha), N)=\{0\}$ for $|\alpha| \leqslant \alpha_{2}$. Now by the corollary to Theorem 4.3 (making $\alpha_{2}$ smaller, if necessary) it follows that $i(\pi(\alpha),\{0\})=i(\pi(-\alpha),\{0\})$. However, $i(\pi(\alpha),\{0\})=\Sigma^{d_{\alpha}}$ and $i(\pi(-\alpha),\{0\})=\Sigma^{d_{-\alpha}}$, where $d_{\alpha}=\operatorname{dim} U_{\alpha}, d_{-\alpha}=\operatorname{dim} U_{-\alpha}$. This contradicts our assumption.

A similar result can be proved for the more general case of a family of NFDE's with stable difference operators. Details are omitted.

EXAMPLE 4.2 (CF. [2, PP. 79-80]). Let $f: \mathbf{R}^{n} \rightarrow \mathbf{R}^{n}$ be locally Lipschitzian.

Consider the ODE

$$
\dot{x}=f(x(t)) \text {. }
$$

Let $\tilde{K} \subset \mathbf{R}^{n}$ be a compact invariant set of (4.1), let $\tilde{N} \subset \mathbf{R}^{n}$ be a bounded isolating neighborhood of $\tilde{K}$, and let $\left\langle\tilde{N}_{1}, \tilde{N}_{2}\right\rangle$ be an index pair in $\tilde{N}$.

We consider the following NFDE (4.2) to be a perturbation of (4.1):

$$
(d / d t) D(\delta) \cdot x_{t}=f\left(x_{1}\left(t-\varepsilon_{1}\right), \ldots, x_{n}\left(t-\varepsilon_{n}\right)\right) .
$$

We assume that $0 \leqslant \varepsilon_{i} \leqslant 1, \delta \in\left(-\delta_{0}, \delta_{0}\right), \delta_{0}>0$, and that $D(\delta): C=$ $C\left([-1,0], \mathbf{R}^{n}\right) \rightarrow \mathbf{R}^{n}$ is a bounded linear operator for each $\delta$, such that the following assumptions are satisfied.

(1) $D(\delta) \cdot \phi$ is continuous (in $(\delta, \phi))$ at $(0, \phi)$, and $D(0) \cdot \phi=\phi(0)$, for every $\phi \in C$.

(2) Every $D(\delta)$ admits the representation

$$
D(\delta) \phi=A(\delta) \phi(0)+\int_{-r}^{0^{-}} d_{\theta} \mu(\theta, \delta) \phi(\theta)
$$

where $A(\delta) \in L\left(\mathbf{R}^{n}, \mathbf{R}^{n}\right)$, det $A(\delta) \neq 0$, the set $\left\{A^{-1}(\delta) \mid \delta \in\left(-\delta_{0}, \delta_{0}\right)\right\}$ is bounded in $L\left(\mathbf{R}^{n}, \mathbf{R}^{n}\right), \mu(\cdot, \delta):[-r, 0] \rightarrow \mathbf{R}^{n}$ is of bounded variation, and $\int_{\alpha}^{0^{-}} d_{\theta}\|\mu(\theta, \delta)\| \rightarrow$ 0 , as $\alpha \rightarrow 0^{-}$, uniformly in $\delta$.

(3) $D(\delta)$ is stable, uniformly in $\delta$, i.e. the constants $a, b$ in the definition of stability (see Example $1.1, \S 1$ ) can be chosen independently of $\delta$.

(2) implies in particular that $D(\delta)$ is atomic at zero. Let $\varepsilon=\left(\varepsilon_{1}, \ldots, \varepsilon_{n}\right)$, $0 \leqslant \varepsilon_{i} \leqslant 1, \delta \in\left(-\delta_{0}, \delta_{0}\right)$, and let $\pi(\delta, \varepsilon)$ be the local semiflow generated by (4.2). Results in [6] imply that, whenever $\left(\delta_{n}, \varepsilon^{n}\right) \rightarrow(0,0)$, then the pair $\left\langle\left\{\pi\left(\delta_{n}, \varepsilon^{n}\right)\right\}, \pi(0,0)\right\rangle$ satisfies the continuity property.

Now let $\nu=\left(\sup _{x \in \tilde{N}}\|x\|\right)+1, \mu=\sup _{\|x\|<\nu}\|f(x)\|$, and consider the following sets:

$$
\begin{aligned}
& K=\{\phi \in C \mid \phi(-r) \in \tilde{K}, \dot{\phi}(\theta) \text { exists and } \dot{\phi}(\theta)=f(\phi(\theta)) \text { for } \theta \in[-r, 0]\}, \\
& N=\{\phi \in C \mid\|\phi\| \leqslant \nu, \phi(0) \in \tilde{N}\}, \\
& N_{1}=\left\{\phi \in C \mid\|\phi\| \leqslant \nu, \phi(0) \in \tilde{N}_{1}\right\}, \\
& N_{2}=\left\{\phi \in C \mid\|\phi\| \leqslant \nu, \phi(0) \in \tilde{N}_{2}\right\} .
\end{aligned}
$$

An easy computation shows that $K$ is an isolated invariant set of $\pi(0,0), N$ is an isolating neighborhood of $K$ (with respect to $\pi(0,0)$ ), and $\left\langle N_{1}, N_{2}\right\rangle$ is an index pair in $N$ (with respect to $\pi(0,0)$, again). 
Moreover, if $\left(\delta_{n}, \varepsilon^{n}\right) \rightarrow(0,0)$, then $\left\langle\left\{\pi\left(\delta_{n}, \varepsilon^{n}\right)\right\}, \pi\right\rangle$ is $N$-admissible.

By making $\delta_{0}$ smaller, if necessary, it follows from Theorem 4.3 that $N$ is an isolating neighborhood for $\pi(\delta, \varepsilon), \delta \in\left(-\delta_{0}, \delta_{0}\right), \varepsilon=\left(\varepsilon_{1}, \ldots, \varepsilon_{n}\right), 0<\varepsilon_{k}<\tilde{\varepsilon}_{0}$.

Let $K(\delta, \varepsilon)=\mathfrak{S}(\pi(\delta, \varepsilon), N)$. Then $i(\pi(\delta, \varepsilon), K(\delta, \varepsilon))=i(\pi(0,0), K)$. Now, $i(\pi(0,0), K)$ is the homotopy type of $N_{1} / N_{2}$. By using the homotopy: $F: N_{1} / N_{2} \times$ $[0,1] \rightarrow N_{1} / N_{2}, F([\phi] ; \sigma)=\left[\phi_{\sigma}\right]$, where $\phi_{\sigma}(\theta)=(1-\sigma) \phi(\theta)+\sigma \phi(0)$, it follows easily that $N_{1} / N_{2}$ and $\tilde{N}_{1} / \tilde{N}_{2}$ have the same homotopy type. Hence the index of $K(\delta, \varepsilon)$, for $(\delta, \varepsilon)$ small, is determined by the ODE (4.1), and one can use certain qualitative properties of (4.1) to obtain statements about (4.2).

Before giving the next example, let us note that the index is "nonnegative" in the following sense: If $K_{1}$ and $K_{2}$ are two disjoint, isolated $\pi$-invariant sets and if $i\left(\pi, K_{1} \cup K_{2}\right)=\overline{0}$, then $i\left(\pi, K_{1}\right)=i\left(\pi, K_{2}\right)=\overline{0}$.

EXAMPLE 4.3 (CF. [2, P. 11]). Let $C_{n}=C\left([-r, 0], \mathbf{R}^{n}\right)$ and suppose that, for every $i=1, \ldots, n, D_{i}: C_{1} \rightarrow \mathbf{R}$ is a stable difference operator, and $f_{i}: C_{1} \rightarrow \mathbf{R}$ is a locally Lipschitzian mapping such that the following assumptions are satisfied:

(1) If $x \in C_{1}$ is a constant function, then $f_{i}(x)=0$ if and only if $x=0$.

(2) If $f_{i}^{\prime}(0)$ is the derivative of $f_{i}$ at zero and $1 \in C_{1}$ is the constant function equal to 1 , then $D_{i} 1 \neq 0$. Furthermore, if $i \neq n$, then also $f_{i}^{\prime}(0) \cdot 1 \neq 0$.

(3) $f_{n}(\phi)=\int_{-r}^{0} \phi^{2}(\theta) d \eta_{n}(\theta)$, where $\eta_{n}:[-r, 0] \rightarrow \mathbf{R}$ is a nondecreasing function.

(4) If $D\left(e^{\lambda}\right)=0$, then re $\lambda \neq 0$.

Consider the following system of equations:

$$
\frac{d}{d t}\left(D_{i}\left(x_{t, i}\right)\right)= \begin{cases}f_{i}\left(x_{t, i+1}\right) & \text { if } i=1, \ldots, n-1, \\ f_{i}\left(x_{t, 1}\right)-\varepsilon & \text { if } i=n .\end{cases}
$$

If $D: C_{n} \rightarrow \mathbf{R}^{n}$ and $f: C_{n} \rightarrow \mathbf{R}^{n}$ are defined by $D(\phi)=\left(D_{1} \phi_{1}, \ldots, D_{n} \phi_{n}\right)^{T}, f(\phi)=$ $\left(f_{1}\left(\phi_{2}\right), \ldots, f_{n}\left(\phi_{1}\right)\right)^{T}$, then $\left(4.3_{\varepsilon}\right)$ takes the form $(d / d t) D x_{t}=f\left(x_{t}\right)-(0, \ldots, \varepsilon)^{T}$; hence $\left(4.3_{e}\right)$ is a NFDE of the type considered in Example 1.1 of $\S 1$.

We shall now prove that under the above assumptions, for every neighborhood $N \subset C_{n}$ of zero there is an $\varepsilon_{0}>0$ such that for every $0<\varepsilon<\varepsilon_{0}$ there is a nonconstant solution $x_{t}=x_{t}(\varepsilon)$ defined for $t \in \mathbf{R}$, such that $x_{t} \in N$ for all $t$.

Proof. We make the following easily proved remarks:

(1) If $D: C_{1} \rightarrow \mathbf{R}$ is stable, $D \cdot 1 \neq 0, x \in C(\mathbf{R}, \mathbf{R}),\left(x_{t}\right)_{t \in \mathbf{R}}$ is precompact, and $D x_{t}=c+d t$ for some constants $c, d \in \mathbf{R}$ and all $t \in \mathbf{R}$, then $d=0$ and $x_{t} \equiv$ const.

(2) If $D: C_{1} \rightarrow \mathbf{R}$ is stable, $y \in C\left(\mathbf{R}^{+}, \mathbf{R}\right), y$ is bounded and $D y_{t}>c+d t$ for some constants $c, d \in \mathbf{R}$ and all $t \in \mathbf{R}^{+}$, then $d \leqslant 0$.

$1^{\circ}$ step. Remark (2) implies that there is no bounded solution of $\left(4.3_{\mathrm{e}}\right)$ for $\varepsilon<0$.

$2^{\circ}$ step. Let $N$ be a closed neighborhood of 0 in $C_{n}$. We can assume that $N$ is bounded and $f$ is bounded on $N$. Let $\pi_{\varepsilon}$ be the local semiflow generated by $\left(4.3_{\varepsilon}\right)$ and $K_{\varepsilon}$ be the largest $\pi_{e}$-invariant set in $N$. By $1^{\circ}$ step, $K_{\varepsilon}=\varnothing$ for $\varepsilon<0$. We will show that $K_{0}=\{0\}$. This implies that there is an $\varepsilon_{0}>0$ such that $K_{e} \subset$ Int $N$ and $i\left(\pi_{\varepsilon}, K_{e}\right)=\overline{0}$ for $|\varepsilon| \leqslant \varepsilon_{0}$. We will also show that for all small positive values of $\varepsilon, N$ contains exactly two constant solutions $\phi_{1}(\varepsilon)$ and $\phi_{2}(\varepsilon)$ of $\left(4.3_{\varepsilon}\right)$, and at least one of them is hyperbolic, say $\phi_{1}(\varepsilon)$. Hence $K_{\varepsilon} \supset\left\{\phi_{1}(\varepsilon), \phi_{2}(\varepsilon)\right\}$. If $K_{\varepsilon}=\left\{\phi_{1}(\varepsilon), \phi_{2}(\varepsilon)\right\}$, then $i\left(\pi_{\varepsilon}, K_{\varepsilon}\right)=\overline{0}$ and the observation preceding this example shows that 
$i\left(\pi_{\varepsilon},\left\{\phi_{1}(\varepsilon)\right\}\right)=\overline{0}$, which contradicts the hyperbolicity of $\phi_{1}(\varepsilon)$. Hence for all small $\varepsilon>0, K_{\varepsilon}$ contains nonconstant elements. This proves our assertion.

$3^{\circ}$ step. $K_{0}=\{0\}$.

Proof. $K_{0}$ is compact and $0 \in K_{0}$. Let $\phi \in K_{0}$ and let $x_{t} \in K_{0}$ be a solution of (4.3 $)$ through $\phi$, defined for $t \in(-\infty, \infty)$. Then $D_{n}\left(x_{t, n}\right)$ is nondecreasing. Hence $D_{n}\left(x_{t, n}\right) \rightarrow \zeta, t \rightarrow-\infty$, and $D_{n}\left(x_{t, n}\right) \rightarrow \eta$ as $t \rightarrow+\infty,-\infty \leqslant \zeta \leqslant \eta \leqslant+\infty$. Let $\alpha$ and $\omega$ be the $\alpha$ - and $\omega$-limit sets of the orbit $\left\{x_{t}\right\}$, respectively. Then $\alpha \cup \omega \subset K_{0}$, and both $\alpha$ and $\omega$ are $\pi_{0}$-invariant. Also the compactness of $K_{0}$ implies that $-\infty<\zeta \leqslant \eta<+\infty$. We show that $\alpha=\omega=\{0\}$. In fact, let $\psi \in \alpha$. Let $y_{t} \in \alpha$ be a solution through $\psi$, defined for $t \in(-\infty, \infty)$. Then $D_{n}\left(y_{t, n}\right)=\zeta$ for $t \in \mathbf{R}$. Hence $y_{t, 1} \equiv 0$ and remark (1) implies that $y_{t, n}=$ constans $=k_{n}$. Hence $(d / d t)\left(D_{n-1}\left(y_{t, n-1}\right)\right)=f_{n-1}\left(k_{n}\right)$. Hence $f_{n-1}\left(k_{n}\right)=0$ by remark (1). This implies that $k_{n}=0$ and $D_{n-1}\left(y_{t, n-1}\right)=$ constans. Arguing analogously, one obtains that $y_{t, n-1} \equiv 0$. Proceeding in this way from $n$ to 1 we see that $y_{t} \equiv 0$. Hence $\psi=0$, which proves $\alpha=\{0\} . \omega=\{0\}$ is proved similarly.

It follows that $\zeta=\eta=0$; hence $D_{n}\left(x_{t, n}\right)=0$ for $t \in \mathbf{R}$. Using the same argument as the one we applied to $y_{t}$ we prove that $x_{t} \equiv 0$, and hence $\phi=0$. This proves our claim.

$4^{\circ}$ step. If $\varepsilon>0$ then there are exactly two constant solutions of $\left(4.3_{\varepsilon}\right)$, namely $\phi_{1}(\varepsilon)=\left(0, \ldots, 0, p_{n} \cdot(\varepsilon)^{1 / 2}\right)^{T}$ and $\phi_{2}(\varepsilon)=\left(0, \ldots, 0,-p_{n} \cdot(\varepsilon)^{1 / 2}\right)^{T}$ where $p_{n}=$ $\left(\eta_{n}(0)-\eta_{n}(-r)\right)^{-1}$. Note that $p_{n} \neq \infty$ by assumption (1).

Let $\sigma_{j}(\varepsilon), j=1,2$, be the spectrum of the linear NFDE obtained by linearizing (4.3) at $\phi_{j}(\varepsilon)$. A simple computation using Theorem 10.1, Chapter 12 in [6] shows that $\lambda \in \sigma_{j}(\varepsilon)$ if and only if

$$
(-1)^{j} \cdot 2 \cdot p_{n} \cdot(\varepsilon)^{1 / 2}\left(\int_{-r}^{0} e^{\lambda \theta} d \eta_{n}(\theta)\right) \cdot \prod_{i=1}^{n-1}\left(f_{i}^{\prime}(0) e^{\lambda \cdot}\right)=\lambda^{n} \cdot \prod_{i=1}^{n}\left(D_{i} e^{\lambda \cdot}\right) .
$$

By assumptions (1) and (2) the function

$$
g(\lambda)=2 \cdot p_{n} \cdot\left(\int_{-r}^{0} e^{\lambda \theta} d \eta_{n}\right) \cdot \prod_{i=1}^{n-1}\left(f_{i}^{\prime}(0) \cdot e^{\lambda \cdot}\right) \cdot\left(\prod_{i=1}^{n}\left(D_{i} e^{\lambda \cdot}\right)\right)^{-1}
$$

is defined and analytic in a neighborhood of $\lambda=0$. Also, by assumption (4), there is a constant $M>0$ such that for every $\varepsilon>0$ and every $\lambda \in \sigma_{1}(\varepsilon) \cup \sigma_{2}(\varepsilon)$ with re $\lambda=0$ it follows that $|\lambda|^{n} \leqslant M p_{n}(\varepsilon)^{1 / 2}$. Let $\delta>0$ be such that $|g(\lambda)-g(0)|<\left(\frac{1}{2}\right)$ $\cdot|g(0)|$, if $|\lambda|<\delta$. Let $0<\varepsilon_{1}<\varepsilon_{0}\left(\right.$ see $2^{\circ}$ step) be such that $M p_{n}(\varepsilon)^{1 / 2}<(\delta)^{1 / n}$ for $0<\varepsilon<\varepsilon_{1}$.

$1^{\circ}$ case. $n$ is even. If $\lambda \in \sigma_{1}(\varepsilon) \cup \sigma_{2}(\varepsilon), 0<\varepsilon \leqslant \varepsilon_{1}$, re $\lambda=0$, then $g(\lambda)$ is real and $\operatorname{sign} g(\lambda)=\operatorname{sign} g(0)$. Hence $\operatorname{sign}(-1)^{j}=\operatorname{sign}(-i)^{n} \cdot \operatorname{sign} g(0)$. Hence either $\phi_{1}(\varepsilon)$ or $\phi_{2}(\varepsilon)$ is hyperbolic.

$2^{\circ}$ case. $n$ is odd. Let $h(\lambda)=\lambda^{n} \cdot(g(\lambda))^{-1}$. Then $h(\lambda)$ is analytic in a neighborhood of $\lambda=0$, and

$$
h(\lambda)=\sum_{k=n}^{\infty} a_{k} \lambda^{k}, \quad a_{n} \neq 0
$$


Suppose $\lambda \in \cup_{0<\varepsilon<\varepsilon_{0}}\left(\sigma_{1}(\varepsilon) \cup \sigma_{2}(\varepsilon)\right)$, re $\lambda=0, \varepsilon_{0}>0$ small. Then, since $\pm p_{n}$. $(\varepsilon)^{1 / 2}$ are real, it follows that $\sum_{k=n ; k \text { odd }}^{\infty} a_{k} \lambda^{k}=0$. Since $a_{n} \neq 0$, it follows that there is only a finite number of such $\lambda$ 's and hence, for all small $\varepsilon>0, \phi_{1}(\varepsilon)$ and $\phi_{2}(\varepsilon)$ are both hyperbolic. This completes the proof of our assertion.

REMARK. If $D_{i} \phi=\phi(0)-\int_{-n}^{0} \phi(\theta) d \mu_{i}(\theta), \operatorname{Var}_{[-s, 0]} \mu_{i} \rightarrow 0$ as $s \rightarrow 0$, and $\mu$ has no singular part, then it follows from Theorem 4.1, Chapter 12 in [6] that assumption (4) in Example 4.3 is satisfied.

To conclude this section, we shall present a result which says that, under certain assumptions, the Alexander-Spanier cohomology module of $N_{1} / N_{2}$ is isomorphic to the cohomology module of a finite-dimensional set.

We assume the reader's familiarity with the Alexander-Spanier cohomology theory and refer to [11] for details.

Let $R$ be a commutative ring and let $G$ be an $R$-module. Consider a topological pair $(X, A)$ and denote by $\bar{H}^{q}(X, A ; G)$ the Alexander-Spanier cohomology module of $(X, A)$ of degree $q$ with coefficients $G$. If $(X, p)$ is a pointed space, define $\bar{H}^{q}(X, p ; G)=\bar{H}^{q}(X,\{p\} ; G)$. If $A=\varnothing$, we write $\bar{H}^{q}(X ; G)=\bar{H}^{q}(X, A ; G)$.

If $X$ is compact and the Hausdorff $\operatorname{dimension} \operatorname{dim}(X \backslash A) \leqslant m<\infty$, then $\bar{H}^{q}(X, A ; G)=0$ for $q>m$.

If $f:(X, A) \rightarrow(Y, B)$ is a morphism, we denote by

$$
f^{*}: \bar{H}^{q}(Y, B ; G) \rightarrow \bar{H}^{q}(X, A ; G)
$$

the induced homomorphism of cohomology modules.

Suppose now that $X$ is a metric space and $A$ is a nonempty closed subset of $X$. Let $p=[A]$ be the distinguished element of $X / A$ and let $\left(U_{\alpha}\right)_{\alpha}$ be the family of all open neighborhoods of $p$ in $X / A$, directed downward by inclusion. Then $\left(\bar{H}^{q}\left(U_{\alpha} ; G\right)\right)_{\alpha}$ together with the corresponding inclusion induced homomorphisms forms a direct system. Let $\lim _{\rightarrow} \bar{H}^{q}\left(U_{\alpha} ; G\right)$ be its direct limit. Now the following theorem holds:

THEOREM 4.4. The inclusion maps $i_{\alpha}:\{p\} \rightarrow U_{\alpha}$ induce an isomorphism $\left\{i_{\alpha}^{*}\right\}$,

$$
\left\{i_{\alpha}^{*}\right\}: \lim _{\rightarrow} \bar{H}^{q}\left(U_{\alpha} ; G\right) \stackrel{\sim}{\rightarrow} \bar{H}^{q}(\{p\} ; G) .
$$

The proof of Theorem 4.4 is analogous to that of Theorem 6.6.2 in [11], with the constant map $f: N \rightarrow\{p\}$ defined on a suitable neighborhood $N$ of $p$.

COROllary. If $X$ is a metric space and $A$ is any closed subset of $X$, then the quotient map $\psi:(X, A) \rightarrow X / A$ induces an isomorphism $\psi^{*}$,

$$
\psi^{*}: \bar{H}^{q}(X / A ; G) \stackrel{\simeq}{\rightarrow} \bar{H}^{q}(X, A ; G) .
$$

Proof. If $A=\varnothing$, then the corollary follows from Lemma 6.4.4 in [11]. If $A \neq \varnothing$, then the corollary is proved exactly like Theorem 6.6 .5 , with the exception that we use Theorem 4.4 above instead of Corollary 6.6.3 from [11].

We can also prove the following extension of the continuity property for the Alexander-Spanier cohomology theory. 
TheOREM 4.5. Let $\left\{\left(X_{\alpha}, A_{\alpha}\right)\right\}_{\alpha}$ be a family of closed pairs in some metric space, directed downward by inclusion. Assume that the following property holds:

(PREC): every net $\left(x_{\alpha}\right)_{\alpha}$ with $x_{\alpha} \in X_{\alpha}$ is precompact.

Let $(X, A)=\left(\cap_{\alpha} X_{\alpha}, \cap_{\alpha} A_{\alpha}\right)$.

Under these assumptions, the inclusion maps $i_{\alpha}:(X, A) \rightarrow\left(X_{\alpha}, A_{\alpha}\right)$ induce an isomorphism $\left\{i_{\alpha}^{*}\right\}$,

$$
\left\{i_{\alpha}^{*}\right\}: \lim _{\rightarrow} \bar{H}^{q}\left(X_{\alpha}, A_{\alpha} ; G\right) \stackrel{\sim}{\rightarrow} \bar{H}^{q}(X, A ; G)
$$

Proof. If $F$ is a closed subset of $X_{\beta}$ for some $\beta$, and $X \cap F=\varnothing$, then (PREC) implies that $X_{\alpha} \cap F=\varnothing$ for some $\alpha$. Now the proof proceeds in exactly the same way as that of Theorem 6.6.6 in [11].

We shall apply Theorem 4.5 to index pairs. Let $N$ be any isolating neighborhood (with respect to $\pi$ ), and $\left(N_{1}, N_{2}\right)$ be an index pair in $N$. We can assume w.l.o.g. that $N_{2} \subset N_{1}$. Suppose that $N$ satisfies (H2) and (H3). Consider the family $\left(X_{t}, A_{t}\right)=$ (Cl $\left.N_{1}^{t}, \mathrm{Cl} N_{1}^{t} \cap N_{2}\right)$.

It follows from (H2) that $\left(X_{t}, A_{t}\right)$ satisfies (PREC). Earlier results in this section imply that all the inclusion induced maps $\hat{e}_{t}: X_{t} / A_{t} \rightarrow N_{1} / N_{2}$ and $\hat{i}_{t}^{s}: X_{s} / A_{s} \rightarrow$ $X_{t} / A_{t}, 0 \leqslant t \leqslant s$, are homotopy equivalences. Hence the corollary of Theorem 4.4 implies that

$$
e_{t}^{*}: \bar{H}^{q}\left(N_{1}, N_{2} ; G\right) \rightarrow \bar{H}^{q}\left(X_{t}, A_{t} ; G\right)
$$

and

$$
i_{t}^{s^{*}}: \bar{H}^{q}\left(X_{t}, A_{t} ; G\right) \rightarrow \bar{H}^{q}\left(X_{s}, A_{s} ; G\right)
$$

are isomorphisms. Now $(X, A)=\left(\cap_{t \geqslant 0} X_{t}, \cap_{t>0} A_{t}\right)=\left(N_{1} \cap A^{-}(N), N_{2} \cap\right.$ $\left.A^{-}(N)\right)$.

Hence the inclusion $i:\left(N_{1} \cap A^{-}(N), N_{2} \cap A^{-}(N)\right) \rightarrow\left(N_{1}, N_{2}\right)$ induces an isomorphism of $\bar{H}^{q}\left(N_{1}, N_{2} ; G\right)$ and $\bar{H}^{q}\left(N_{1} \cap A^{-}(N), N_{2} \cap A^{-}(N) ; G\right)$.

Summarizing, we obtain the following theorem:

THEOREM 4.6. Let $N$ be an isolating neighborhood (for $\pi$ ), satisfying (H2) and (H3), and let $\left(N_{1} / N_{2}\right)$ be an index pair in $N$.

Then there is an inclusion induced isomorphism

$$
i^{*}: \bar{H}^{q}\left(N_{1}, N_{2} ; G\right) \stackrel{\sim}{\rightarrow} \bar{H}^{q}\left(N_{1} \cap A^{-}(N), N_{1} \cap A^{-}(N) ; G\right) .
$$

Hence

$$
\hat{i}^{*}: \bar{H}^{q}\left(N_{1} / N_{2} ; G\right) \stackrel{\sim}{\rightarrow} \bar{H}^{q}\left(N_{1} \cap A^{-}(N) / N_{2} \cap A^{-}(N) ; G\right) .
$$

In particular, if $\operatorname{dim}\left(N_{1} \cap A^{-}(N)\right) \leqslant m<\infty$, then

$$
\bar{H}^{q}\left(N_{1}, N_{2} ; G\right)=\bar{H}^{q}\left(N_{1} / N_{2} ; G\right)=0 \quad \text { for } q>m .
$$

The importance of Theorem 4.6 is seen more clearly if we invoke the fact that, under some additional assumptions, the set $A^{-}(N)$ has finite topological dimension. We shall now quote the corresponding theorem due to R. Mañé [9], who 
generalized an earlier result of $\mathbf{J}$. Mallet-Paret [8], and, at the same time, greatly simplified its proof. Let us first recall the following concept:

Definition 4.1 (CF. [9]). Let $K$ be a compact metric space. Define $N(r, K)$ to be the smallest number of open balls of radius $r$ required to cover $K$.

Set

$$
c(K)=\limsup _{r \rightarrow \infty} \frac{\log N(r, K)}{\log (1 / r)} .
$$

$c(K)$ is called the limit capacity of $K$.

It is well known that $\operatorname{dim}(K) \leqslant c(K)$, but there are cases where $\operatorname{dim}(K)<c(K)$ $=\infty$.

THEOREM 4.7 ([9], CF. Also [8]). Let $E$ be a Banach space, $U \subset E$ an open set and $T: U \rightarrow E$ a $C^{1}$-map. Let $\Lambda \subset E$ be a compact set such that $T(\Lambda) \supset \Lambda$. Suppose that for every $x \in \Lambda$, the derivative $D T(x)$ is the sum of two linear operators $T_{1}, T_{2}$ such that $T_{1}$ is a contraction and $T_{2}$ is compact.

Then $c(\Lambda)<\infty$. In particular, $\Lambda$ has finite Hausdorff dimension.

COROllary. Let $X$ be an open subset of a Banach space $E$, and let $\pi$ be a local semiflow in $X$. If $N$ is a closed subset of $X$ satisfying (H2) and (H3), and if, for some $t_{0}$, the map $T$ defined by $x \rightarrow x \pi t_{0}$ is of class $C^{1}$ and the derivatives of $T$ at every point of $A^{-}(N)$ is the sum of a contraction and a compact operator, then $c\left(A^{-}(N)\right)$ $<\infty$.

Hence, under the assumptions of Theorem 4.6 and the corollary of Theorem 4.7, the set $N_{1} / N_{2}$ behaves cohomologically like a finite-dimensional set. In particular, only a finite number of the modules $\bar{H}^{q}\left(N_{1} / N_{2} ; G\right)$ are nontrivial.

The assumptions of the corollary concerning $T$ are satisfied if $\pi$ is the local semiflow generated as in Examples 1.1 and 1.2 by an NFDE with a stable difference operator or by a semilinear parabolic PDE, under the additional assumption that the right-hand side of the equation is continuously differentiable and completely continuous. This follows from results in [6] and [7].

ACKNOWLEDGement. I wish to express my sincere appreciation to Professor Jack K. Hale. His advice and inspiration have led to a significant improvement of the original version of this paper.

\section{REFERENCES}

1. R. Churchill, Isolated invariant sets in compact metric spaces, J. Differential Equations 12 (1972), 330-352.

2. C. C. Conley, Isolated invariant sets and the Morse index, CBMS Regional Conf. Ser. Math., no. 38, Amer. Math. Soc., Providence, R. I., 1978.

3. C. C. Conley and R. W. Easton, Isolated invariant sets and isolating blocks, Trans. Amer. Math. Soc. 158 (1971), 35-61.

4. K. Deimling, Nichtlineare Gleichungen und Abbildungsgrade, Springer-Verlag, Berlin and New York, 1974.

5. A. Friedman, Partial differential equations, Holt, Rinehart and Winston, New York, 1969.

6. J. K. Hale, Theory of functional differential equations, Springer-Verlag, Berlin and New York, 1977.

7. D. Henry, Geometric theory of semilinear parabolic equations, Lecture Notes, University of Kentucky, 1978. 
8. J. Mallet-Paret, Negatively invariant sets of compact maps and an extension of a theorem of Cartwright, J. Differential Equations 16 (1976), 331-348.

9. R. Mañe, On the dimension of the compact invariant sets of certain nonlinear maps (preprint).

10. J. T. Montgomery, Cohomology of isolated invariant sets under perturbations, J. Differential Equations 13 (1973), 257-299.

11. E. H. Spanier, Algebraic topology, McGraw-Hill, New York, 1966.

12. F. W. Wilson, Jr., Smoothing derivatives of functions and applications, Trans. Amer. Math. Soc. 139 (1969), 413-428.

13. F. W. Wilson Jr. and J. A. Yorke, Lyapunov functions and isolating blocks, J. Differential Equations 13 (1973), 106-123.

14. N. Bhatia and O. Hajek, Local semi-dynamical systems, Lecture Notes in Math., vol. 90 , Springer-Verlag, Berlin and New York, 1969.

15. R. D. Nussbaum, The fixed point index for local condensing maps, Ann. Mat. Pura Appl. (4) 89 (1971), 217-258.

16. J. D. Monk, Introduction to set theory, McGraw-Hill, New York, 1969.

Lefschetz Center for Dynamical Systems, Division of Applied Mathematics, Brown UniverSity, Providence, Rhode Island 02912 